\title{
Anatomía de un problema público: la ausencia de la prevención de enfermedades crónico degenerativas en el Presupuesto de Egresos de la Federación, 2010-2015
}

\author{
Gabriela Tapia Téllez \\ Universidad Autónoma de la Ciudad de México \\ Jaime Hernández Colorado \\ Centro de Investigación y Docencia Económicas
}

Recepción: 14 de noviembre de 2016 Aceptación: 26 de abril de 2017

Resumen Este artículo analiza la política de salud pública federal en México en tres aspectos: 1) los programas presupuestarios dirigidos al ámbito de la salud y sus asignaciones (Ramo 12, Secretaría de Salud; Ramo 50, Imss; Ramo 51, Issste, del Presupuesto de Egresos de la Federación [PEF], SHCP); 2) la situación actual de México en materia epidemiológica por edad, sexo, región geográfica, entidad y grupo vulnerable, y 3) las proyecciones demográficas y su relación con los problemas epidemiológicos identificados. La base metodológica de la investigación es el análisis de política pública como proceso de solución de problemas e hipótesis causal (Aguilar, 2010, p. 32). El principal argumento de este artículo es que no se observa que exista una relación causal de los principales problemas epidemiológicos y su prevención con la distribución del presupuesto vía programas aprobados en el PEF entre 2010 y 2015. La ausencia causal identificada es significativa en materia de finanzas públicas, ya que pone en riesgo la sostenibilidad del sistema de salud en México, sobre todo si las tendencias demográficas y la evolución de las enfermedades crónico degenerativas se mantiene.

PALABRAS CLAVE: salud pública, política pública, prevención, presupuesto, programas presupuestarios, México.

Abstract The paper analyzes health policy in Mexico in three aspects: 1) budget programs on health and its allocations (Secretaría de Salud, IMSS, ISSSTE) on the Federal Budget; 2) current epidemiological situation by age, sex, geographical region, locality and vulnerable group; 3 ) demographic pro- 
jections and its relation with the epidemiological problems identified. The methodological base of this paper is public policy analysis as a process of problem solution and causal hypothesis (Aguilar, 2010, p. 32). The main argument of this paper is that cannot observe any causal relation between the main epidemiological problems and its prevention through the distribution of the Federal Budget and its approved health programs during 2010-2015. This causal absence is significant in financial terms, as it is risky for the sustainability of the health system in Mexico, more if we consider that demographic trends and the evolution of cronic-degenerative diseases persist.

KEY WORDS: public health, public policy, prevention, budget, budget programs, Mexico.

\section{Introducción}

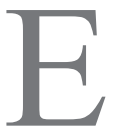

n este artículo se analiza la política de salud pública federal en México en tres aspectos. En primer lugar, los programas presupuestarios dirigidos al ámbito de la salud y sus respectivas asignaciones (Ramo 12, Secretaría de Salud; Ramo 50, IMss, y Ramo 51, Issste, del Presupuesto de Egresos de la Federación, SHCP). En segundo término, la situación actual de México en materia epidemiológica por edad, sexo, región geográfica, entidad y grupo vulnerable. Y, finalmente, en tercer lugar, las proyecciones demográficas y su relación con los problemas epidemiológicos identificados. La base metodológica de esta investigación es el análisis de política pública como proceso de solución de problemas e hipótesis causal (Aguilar, 2010, p.32). El principal argumento del artículo es que no se observa una relación causal entre los principales problemas epidemiológicos, su prevención y la distribución del presupuesto dentro de los ramos dirigidos a la atención de la salud en México (ramos 12, 50 y 51). Esta ausencia pone en riesgo la sostenibilidad del sistema de salud en el país a futuro, dadas las proyecciones demográficas y la evolución de las enfermedades crónico degenerativas.

El análisis de política pública, como proceso de solución de problemas e hipótesis causal parte de una situación social indeseable que se identifica como objeto de intervención gubernamental (Aguilar, 2010, p. 32). En sentido instrumental, la política pública tiene la característica de dirigirse a solucionar todas o la mayor parte de las causales que originan el problema identificado. En este caso, la situación social indeseable está compuesta por los principales problemas epidemiológicos que existen en el país. Nuestra propuesta es que la respuesta que puede disminuir en mayor medida el problema público identificado debería hallarse en el 
conjunto de los programas que integran los ramos presupuestales analizados, pues su objetivo es velar por la salud pública. Esto supone una construcción conceptual y epistemológica que permita entender cómo y por qué, ante los problemas de salud pública identificados, la intervención gubernamental, a través de los programas de los ramos presupuestales analizados, debería ser un intento de solucionarlos. Así, observamos si existe o no relación causal entre las distintas situaciones sociales (epidemiológicas) indeseables y la acción gubernamental mediante el presupuesto federal, en concreto los ramos mencionados. ${ }^{1}$ La evidencia empírica señala que no es así mientras que, como ya dijimos, la opción que podría atacar en buena medida los aspectos sustantivos del problema público identificado es la prevención. De nuevo, como mostraremos en el análisis, la prevención - como estrategia en la política de salud pública - está ausente del Presupuesto de Egresos de la Federación.

El presente artículo se integra de cuatro secciones. En la primera se analizan los principales problemas epidemiológicos y sus causales por distribución, en la segunda se describen las proyecciones demográficas y sus implicaciones para el gasto en salud, en la tercera se analizan los ramos 12, 50 y 51 en relación con los problemas epidemiológicos y sus causales por distribución; finalmente, en la cuarta se concluye con la presentación de algunas recomendaciones breves de política pública que se derivan del análisis. En suma, en su conjunto, el estudio tiene como objetivo demostrar que ciertos padecimientos y su evolución forzarán tarde o temprano a modificar la estructura del gasto en salud, pues hoy no se sostiene la idea de que el gasto en los aspectos estudiados esté atacando la estructura epidemiológica y previniendo un aumento de sus padecimientos principales. Nuestra idea, que atraviesa las sugerencias de política pública de la parte final del artículo, es que tales cambios en la planeación y asignación de recursos deberían preverse, y principalmente intentar evitarlos por medio de inversión en términos preventivos. Es decir, cambiar de inmediato la estructura del gasto en los ramos especializados en salud y evitar que los problemas epidemiológicos del país alcancen proporciones catastróficas.

\section{Principales problemas epidemiológicos en México y sus causales por distribución}

El análisis de esta sección se centra en los principales problemas epidemiológicos en México y su distribución por edad, sexo, entidad federativa, nivel socioeconómico y grupo de vulnerabilidad. Lo anterior, con dos objetivos: 1) caracterizar

Es importante aclarar que en política pública existen muchos otros marcos analíticos, que no serán abordados en esta investigación aun cuando también son relevantes en materia de salud pública. Por ejemplo, marcos institucionales, organizacionales, de gestión pública y sistémicos, entre otros. Además, en materia de salud pública hay muchos problemas, aparte de los epidemiológicos, que no son contemplados en este análisis. 
puntualmente los problemas epidemiológicos, sus causales y las poblaciones que los padecen o podrían padecerlos (población objetivo), y 2) caracterizar la relación que existe entre los programas presupuestales y las causales de los principales problemas epidemiológicos.

El primer problema epidemiológico identificado es la inseguridad alimentaria - relacionada directamente con la desnutrición-que padecen principalmente los niños, es decir, la población de 0 a 14 años, según el Instituto Nacional de Geografía y Estadística (INEGI). En el ámbito nacional, de los 112.3 millones de habitantes que contabilizó el Censo General de Población y Vivienda 2010, 32.5 millones tienen entre 0 y 14 años de edad, lo que representa $28.9 \%$ de la población; esto significa que casi tres de cada diez habitantes del país son niños (INEGI, 2014, p.10). Aunque se ha documentado una disminución en la prevalencia de los diversos tipos de desnutrición infantil a lo largo de 24 años, de acuerdo con las encuestas nacionales de nutrición, la prevalencia de desnutrición crónica (manifestada en baja estatura, por ejemplo) persiste en $13.6 \%$ de los preescolares, lo que representa casi 1.5 millones de menores de cinco años (Cuevas Nasu, Rivera Dommarco, Shamah Levy, Mundo Rosas \& Méndez Gómez, 2014, p. 548). Además, cuando se analiza la desnutrición por estrato económico se observa que es sumamente elevada la prevalencia en los grupos sociales con mayores desventajas; por ejemplo, en la población indígena la cifra de desnutrición crónica casi se triplica pues alcanza 33 \% (Cuevas Nasu et al., 2014, p. 548).

El problema de la inseguridad alimentaria es relevante desde el punto de vista de política pública si se tiene en cuenta que tres de cada cuatro hogares mexicanos con menores de cinco años viven con este problema y uno de cada tres presenta inseguridad alimentaria moderada o severa (Cuevas Nasu et al., 2014, p. 552). Estudios recientes muestran un aumento del riesgo de desnutrición crónica. Este riesgo es $42 \%$ mayor en menores de cinco años que viven en hogares con inseguridad alimentaria severa que en aquellos que viven en situación de seguridad alimentaria (Cuevas Nasu et al., 2014, p. 552). Sobre este problema, es importante resaltar que los hogares indígenas y de localidades rurales concentran la mayor prevalencia de inseguridad alimentaria moderada o severa, así como mayores proporciones de niños con desnutrición crónica (Cuevas Nasu et al., 2014, p. 552).

La inseguridad alimentaria también se asocia a problemas como la anemia, que en menores de dos años alcanza a casi $40 \%$, y en niños de 2 a 5 años a casi 20 $\%$. Por otra parte, uno de cada cinco niños menores de cinco años que viven en hogares con estas características presenta sobrepeso (9.7 \%). En los hogares con inseguridad alimentaria moderada y severa habitan hasta $30 \%$ de escolares con sobrepeso y obesidad (Shamah-Levy, Mundo Rosas \& Rivera Dommarco, 2014, p. 582). La obesidad se debe a que la población en pobreza satisface sus necesidades de consumo de calorías ingiriendo alimentos de bajo costo, altos en energía pero 
con un contenido deficiente de micronutrientes. Es cada vez más frecuente que los hogares accedan a alimentos de alta densidad energética y menor costo, lo que trae como consecuencia ganancias importantes en el peso corporal pero con deficiencia de micronutrientes (Shamah Levy et al., 2014, p. 584). A esto se suma la propensión del mercado de alimentos tradicionales en México a ofrecer productos con una carga calórica excesiva y, en general, a malas prácticas en la producción de ellos, como el uso de grasas recicladas y la optimización de costos reduciendo la calidad de los insumos. No deben obviarse causas como "el descenso en la actividad física originado por los modelos de urbanización y el sedentarismo" ni los factores genéticos (Shamah Levy et al., 2015, p. 4). Sobra señalar que a la tendencia al aumento en la obesidad y el sobrepeso en la población infantil se le debería considerar, por su importancia, un problema de mayor magnitud que el de la desnutrición, sobre todo por sus consecuencias de mediano y largo plazo (Masud Yunes, Barrios Reyes \& Duarte Ortuño, 2009, pp. 64-65).

México ocupa el cuarto lugar mundial en prevalencia de obesidad infantil con aproximadamente $28.1 \%$ en niños y 29 \% en niñas (Saldívar Cerón, Garmendia Ramírez, Rocha Acevedo \& Pérez Rodríguez, 2015). Según la Encuesta Nacional de Salud y Nutrición 2012 (ensanut 2012), México también presenta una prevalencia de sobrepeso y obesidad en escolares de cinco a once años de $19.8 \%$ y $14.6 \%$, respectivamente, lo que da 34.4 \% en total (Saldívar Cerón et al., 2015, p. 56). Un análisis de cómo se distribuye la obesidad entre las entidades federativas del país indica que los estados con mayor prevalencia de ella en niños de cinco a once años son: Campeche, Baja California, Yucatán, Tamaulipas, Colima y Nuevo León (ENSANUt 2012), mientras que los de menor prevalencia son: Chiapas, Oaxaca, Tlaxcala y Zacatecas (ENSANUT 2012). Un niño con sobrepeso posiblemente será un adulto con sobrepeso y podría tener mayores probabilidades de padecer diabetes mellitus o alguna otra patología asociada al sobrepeso.

Respecto a las causas de muerte, la desigualdad se hace patente de diversas formas. Por ejemplo, la tasa de mortalidad en Guerrero de menores de cinco años es de 25.3 por cada 1,000, mientras que en Nuevo León es de solo 13.1. Por otra parte, la tasa de mortalidad por desnutrición en menores de cinco años es 24 veces mayor en Oaxaca (19.6 por cada 100,000) que en Nuevo León (Lozano et al., 2013, p. 583).

El segundo grupo poblacional en el que nos centramos es el de jóvenes. Este segmento comprende, según el INEGI, de los 15 a los 29 años. México es actualmente un país de jóvenes, la transición demográfica presenta el momento actual con el mayor número de ellos. El Censo General de Población y Vivienda 2010 contabilizó 29.7 millones de jóvenes, lo que representa $26.4 \%$ de la población total del país (INEGI, 2014, p.3). En la juventud, casi la totalidad de las muertes en ambos sexos son causadas, en primer lugar, por homicidios; en segundo, por accidentes de vehículo, y en tercero, por suicidios (Conapo, 2012). Es importante diferenciar las 
tasas por sexo para cada causal, pues en esta etapa se observa un problema mucho mayor para los hombres que para las mujeres.

En México, la mortalidad a causa del suicidio se ha incrementado en los últimos cuarenta años (Borges, Orozco \& Medina Mora, 2012). De acuerdo con datos de la Secretaría de Salud, el suicidio aumentó 215 \% entre 1970 y 1998 (Mondragón, Borges \& Gutiérrez, 2001). El 40.8\% ocurre en jóvenes de 15 a 29 años, entre ellos la tasa es de 7.5 suicidios por cada 100,000 jóvenes. Además, es importante tener en cuenta que, del total de suicidios ocurridos en 2013, 81.7 \% correspondieron a hombres y $18.2 \%$ a mujeres (INEGI, 2015). Si analizamos los suicidios por entidad federativa, en 2013, Aguascalientes, Quintana Roo y Campeche fueron las entidades que presentaron mayor tasa de ellos con $9.2,8.8$ y 8.5 por cada 100,000 habitantes, respectivamente, en tanto que Guerrero (2.7), Oaxaca (3) y Tlaxcala (3.1) presentaron las tasas más bajas (INEGI, 2015).

CUADRO 1. Principales problemas epidemiológicos en niños y sus consecuencias

\begin{tabular}{|c|c|c|}
\hline $\begin{array}{l}\text { Principales problemas } \\
\text { epidemiológicos }\end{array}$ & $\begin{array}{l}\text { Principales } \\
\text { afectados }\end{array}$ & $\begin{array}{c}\text { Consecuencias a mediano } \\
\text { y largo plazo }\end{array}$ \\
\hline 1. Sobrepeso & $\begin{array}{l}\text { 1.Estratos sociales bajos } \\
\text { Entidades } \\
\text { 1. Campeche } \\
\text { 2. Baja California } \\
\text { 3. Yucatán } \\
\text { 4. Tamaulipas } \\
\text { 5. Colima } \\
\text { 6. Nuevo León }\end{array}$ & $\begin{array}{l}\text { Adulto con sobrepeso } \\
\text { Mayores probabilidades de diabetes } \\
\text { mellitus } \\
\text { Patologías asociadas al sobrepeso: } \\
\text { enfermedades crónico degenerativas }\end{array}$ \\
\hline 2. Inseguridad alimentaria & $\begin{array}{l}\text { 1. Estratos sociales bajos } \\
\text { 2. Población indígena } \\
\text { 3. Hogares ubicados en zona } \\
\text { rurales }\end{array}$ & $\begin{array}{l}\text { 1. Anemia } \\
\text { 2. Desnutrición } \\
\text { 3. Baja estatura } \\
\text { 4. Sobrepeso } \\
\text { 5. Obesidad }\end{array}$ \\
\hline
\end{tabular}

Fuente: elaboración propia con base en el análisis de la sección.

En el caso de los accidentes automovilísticos, la principal causa es el alcohol, y la segunda el exceso de velocidad. En el ámbito nacional, durante 2012, $11.6 \%$ de los jóvenes reportaron haber tenido un accidente de tránsito bajo los efectos del alcohol (INEGI, 2014). Como en el caso del suicidio, el consumo de alcohol —que aquí vinculamos con las muertes por accidente de tránsito - tiene una incidencia superior en hombres que en mujeres. En la población femenina estas dos variables presentan cifras claramente inferiores en 2002 (29.6\% y $20.9 \%$, respectivamente), pero han tenido un aumento considerable que ha reducido la brecha con la población masculina, pues en 2011 alcanzaron las cifras respectivas de 39.7 \% y $28.1 \%$ ). 
Aunque se debe considerar que el consumo de alcohol no implica la manifestación del alcoholismo, el inicio de su consumo a edades tempranas puede desarrollar proclividad al consumo excesivo en el largo plazo pues, por un lado, la permisividad en el entorno familiar sienta las bases para considerar que el consumo de alcohol es socialmente aceptable y, por otro, el desarrollo de la persona durante la adolescencia - con las consecuencias sociales que conlleva- refuerza la idea de que es normal este consumo. El hecho de que los jóvenes consuman bebidas embriagantes probablemente será causa de alcoholismo a mediano o largo plazo (González \& Alcalá, 2006, pp. 2-3; ENADIC 2011; Pons \& Berjano, 1999, pp. 63-96).

FIGURA 1. Principal causa de los problemas de salud pública en jóvenes y sus consecuencias

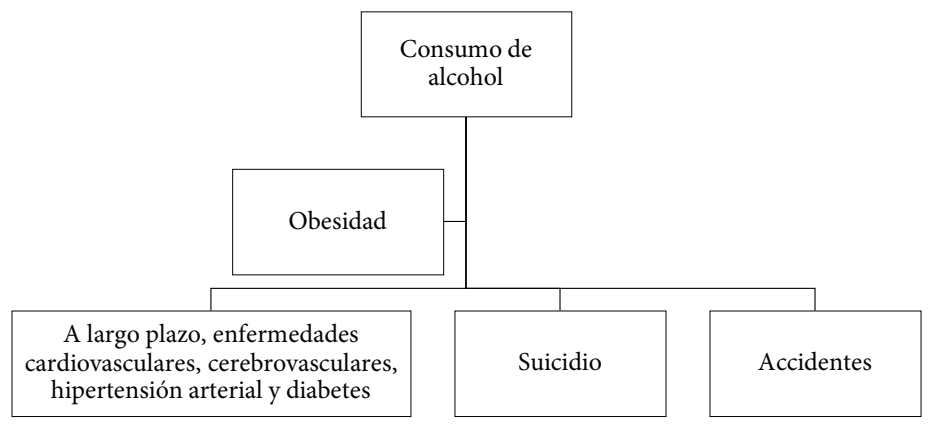

Fuente: Elaboración propia con base en el análisis de la sección.

Otro problema que se identifica en el grupo poblacional de los jóvenes es el sobrepeso. De acuerdo con los resultados de la ENSANUT 2012, 35 \% de ellos tienen sobrepeso u obesidad. Adicionalmente, más de uno de cada cinco adolescentes lo tiene y uno de cada diez presenta obesidad (ENSANUT, 2012, p.169). La obesidad, como veremos más adelante, también está relacionada con el consumo de alcohol $y$, posteriormente, origina la diabetes mellitus. Es preciso destacar que la obesidad en edades tempranas es un factor de riesgo importante para el desarrollo de ciertas patologías en la edad adulta. Por ejemplo, es un factor de riesgo de enfermedades cardiovasculares, cerebrovasculares, hipertensión arterial y diabetes (Campos Nonato, Barquera \& Aguilar, 2012, pp. 328-337).

Siguiendo con el análisis del perfil epidemiológico del país, es necesario reparar en los problemas asociados al grupo de población identificado como adultos, que corresponde a las personas de entre 30 y 64 años de edad. En el caso de los adultos hombres, tres grupos de enfermedades concentran la mitad de los años de vida asociados con discapacidad (AVD): los trastornos mentales (25\%), los problemas musculoesqueléticos (19\%) y los problemas neurológicos (7 \%) (Lozano et al., 2013, p. 583). 
Las cuatro principales causas de muerte en adultos hombres entre 30 y 59 años de edad son: diabetes mellitus, cirrosis y otras enfermedades crónicas del hígado, homicidios y enfermedades isquémicas del corazón (Conapo, 2012). En el caso de las mujeres adultas, la mitad de los AVD se concentran en las enfermedades mentales $(23 \%)$ y musculoesqueléticas ( $23 \%)$, mientras que ocupan los primeros cuatro lugares como causas de muerte entre estas edades la diabetes mellitus, las enfermedades isquémicas del corazón, el cáncer de mama y la cirrosis y otras enfermedades crónicas del hígado. Es importante tener en cuenta que estas enfermedades - tanto las que sufren los hombres como las que padece la población femenina- se encuentran entre las principales causas de muerte en México (ops, 2012, p.517).

Como podemos ver, hombres y mujeres comparten la diabetes mellitus, las enfermedades isquémicas del corazón, la cirrosis y otras enfermedades del hígado como causales de muerte, aunque en diferentes grados, como expondremos a continuación. Por otro lado, cuando analizamos las enfermedades actuales en ambos sexos, los trastornos mentales ocupan el primer lugar y los musculoesqueléticos el segundo.

En el caso de los adultos, del total de esta población en México, 9.17 \% reportó tener un diagnóstico previo de diabetes por un médico, lo que equivale a $6.4 \mathrm{mi}$ llones de personas. Por estado, los cuatro que más padecen esta enfermedad son el Distrito Federal, Nuevo León, Veracruz y el Estado de México. Es importante resaltar que entidades con mayor marginación, como Chiapas y Oaxaca, tienen porcentajes menores en casi tres puntos porcentuales (Hernández Ávila, Gutiérrez \& Reynoso Noverón, 2013, p. 131). Por sexo, los diabéticos fueron $8.6 \%$ de los hombres y $9.67 \%$ de las mujeres, lo que equivale a 2.84 millones de ellos y 3.56 millones de ellas.

CUADRO 2. Principales causas de muerte de adultos, hombres y mujeres

Hombres

1. Diabetes mellitus

2. Cirrosis

3. Enfermedades crónicas del hígado

4. Enfermedades isquémicas del corazón
Mujeres

1. Diabetes mellitus

2. Enfermedades isquémicas del corazón

3. Cáncer de mama

4. Cirrosis y otras enfermedades crónicas del hígado

Fuente: Elaboración propia con base en Conapo (2012).

El análisis por entidad y por sexo revela que, en el caso de los hombres, las entidades con mayor proporción de individuos con diagnóstico de diabetes son: Distrito Federal (12.7\%), Estado de México (11.5\%) y Veracruz (10.7 \%); en tanto que, para las mujeres, las entidades con mayor proporción de personas con diagnóstico de diabetes son: Nuevo León (15.5 \%), Tamaulipas (12.8 \%) y Distrito Federal (11.9 
\%) (Hernández Ávila et al., 2013, p. 131). En términos de las diferencias por nivel socioeconómico, para los cinco quintiles de ingreso se observa el incremento con la edad en la proporción de personas con diagnóstico previo, tanto hombres como mujeres. En general, se encontraron proporciones menores entre las personas del primer quintil (menor nivel), que en todos los casos presentan proporciones menores que el promedio para el grupo de edad y sexo, en tanto que, en todos los casos, las personas en el quinto quintil (mayor nivel) presentan proporciones de diagnóstico de diabetes mayores que el promedio del grupo de edad y sexo. Es necesario tener cuidado con la interpretación de estos resultados porque es posible que una parte de la diferencia esté relacionada con una menor proporción de diagnóstico y no con una menor prevalencia de diabetes (Hernández Ávila et al., 2013, p. 132). Pese a las diferencias por sexo en los datos, la diabetes en México es la principal causa de muerte. De acuerdo con los datos del informe Salud en las Américas, la diabetes sacarina (mellitus) se ha mantenido desde 2005 como la causa de muerte número uno, con un aumento continuado en la incidencia de casos (ops, 2012, p. 517).

La cirrosis, como mencionamos, es un problema de la población adulta relacionado directamente con el consumo de alcohol. Entre 2000 y 2012 se observó un aumento en el porcentaje de adultos que lo consumen; pasó de $39.7 \%$ en 2000 a 34.1 $\%$ en 2006 y $53.9 \%$ en 2012, lo que implica una diferencia estadísticamente significativa. Entre los hombres, el consumo pasó de $56.1 \%$ en 2000 a $67.8 \%$ en 2012 . El cambio también fue claro entre las mujeres, pues de $24.3 \%$ de ellas que consumía alcohol en 2000 este indicador se elevó en 2012 a 41.3 \% (Guerrero López, Muños Hernández, Sáenz de Miera Juárez, Pérez Núñez \& Reynales Shigematsu, 2013, p. 284). Lo anterior resulta de suma importancia porque la mortalidad atribuible a enfermedades hepáticas, relacionadas con el consumo de alcohol, presenta un aumento constante entre 2000 y 2010 y se sitúa en 18,192 en promedio por año. Es importante tener en cuenta, sin embargo, que la mortalidad en hombres por esas causas es casi cinco veces mayor que la mortalidad en mujeres: 14,868 muertes anuales en promedio para los hombres y 3,322 para las mujeres (Guerrero López et al., 2013, 285).

Pasemos ahora al análisis del cáncer en México. Las tasas de mortalidad anual por cáncer para todas las edades, en 2010 y 2011, mantuvieron un comportamiento similar: 77.7 y 71.8 por cada 100,000 en hombres y 78.5 y 72.8 por $100,000 \mathrm{en} \mathrm{mu-}$ jeres, respectivamente. Sin embargo, el riesgo de morir por cáncer entre las mujeres fue $44 \%$ mayor que entre los hombres, principalmente en el grupo de 35 a 64 años (Torres Sánchez, Rojas Martínez, Escamilla Núñez, Vara Salazar \& Lazcano Ponce, 2014, p. 477). En el caso del cáncer de mama, a partir de 2004, como consecuencia de la reducción de la mortalidad por cáncer de pulmón (-3.2 \% en hombres y -1.8 $\%$ en mujeres), estómago ( $-2.1 \%$ en hombres y $-2.4 \%$ en mujeres) y cérvix ( $-4.7 \%)$, se observó una disminución significativa $(\sim 1 \%$ anual $)$ en la mortalidad por cáncer 
en general tanto en todas las edades como en el grupo de 35 a 64 años para ambos sexos. Sin embargo, la mortalidad por otros tipos de cáncer, como el de mama y el de ovario, en las mujeres, o el de próstata, en los hombres, mostró un aumento sostenido (Torres Sánchez et al., 2014, p. 473).

Es interesante resaltar que, al igual que el resto de las enfermedades crónico degenerativas que hemos analizado, el cáncer está asociado al consumo de alcohol. Su consumo es un factor de riesgo para diferentes tipos de cáncer, entre ellos el tumor maligno de hígado y el cáncer de colon y recto. Además, hay que tener en cuenta el papel que pueden desempeñar los cambios dietéticos en probabilidad de incidencia de cáncer. México se encuentra entre los países con más altos niveles de consumo de alcohol en el mundo y este ha mostrado un incremento sostenido durante los últimos cincuenta años. El principal determinante del cáncer de hígado es la cirrosis hepática, asociada al consumo de alcohol. Ocupa el segundo lugar la infección por el virus de la hepatitis, otra patología común en el país, pues las condiciones de precaución en el ámbito sanitario, por ejemplo en las transfusiones sanguíneas, cambiaron hace unas cuantas décadas (Torres Sánchez et al., 2014, p. 487).

El cáncer de mama es la tercera causa de muerte entre las mujeres en México. Algunos de sus factores de riesgo son las dietas hiperenergéticas, que tienen como consecuencia sobrepeso u obesidad. Estudios muy recientes recolectaron datos relacionados con el estado nutricional de pacientes con cáncer de mama y encontraron que casi la totalidad de la muestra evaluada (86\%) presentaba sobrepeso u obesidad. De igual manera, se encontró que las pacientes evaluadas (97\%) en este proyecto se caracterizaron por presentar una distribución de grasa androide, que se refleja en una circunferencia abdominal mayor que la recomendada para la población mexicana. Estas condiciones, propias del síndrome metabólico (o síndrome X), tienen incidencia en el desarrollo de otras patologías, como la diabetes o la hipertensión. En México, una de cada dos personas padecen el síndrome metabólico, y la prevalencia es más elevada en mujeres que en hombres (Cordero \& Pinto, 2014, p. 17). Esto es importante porque las anormalidades metabólicas y endocrinas características de la obesidad son más pronunciadas en personas con distribución androide, en quienes la grasa tiende a acumularse en el abdomen. De hecho, se ha establecido que la distribución androide agrava el riesgo de desarrollar cáncer de mama, ya presente por la obesidad, y que existe asociación positiva entre la adiposidad abdominal —-definida tanto por índice de cintura cadera (ICC) como por circunferencia abdominal- y el riesgo de desarrollar carcinoma mamario (Gutiérrez, Autrique \& Ceballos, 2012, p. 189).

Los individuos del grupo de población conocido como adultos mayores corresponden, según el INEGI, a la población mayor de 64 años y representan $6.2 \%$ de la población total en 2010 (INEGI, 2014, p. 14). En este rango de edad, los tres padecimientos con mayor autorreporte de diagnóstico médico, en las personas del 
sexo masculino, fueron: hipertensión (40.0\%), diabetes (24.3\%) e hipercolesterolemia $(20.4 \%)$. En relación con padecimientos mentales, uno de cada seis presentó síntomas depresivos significativos (17.6\%), algo más de $7 \%$ deterioro cognitivo y alrededor de $8 \%$ demencia. En relación con la pérdida de autonomía, $26.9 \%$ presentó dificultad para realizar al menos una actividad básica de la vida diaria, y 24.6 $\%$ para realizar al menos una actividad instrumental de la vida cotidiana. En ambas condiciones las prevalencias se incrementan a medida que aumenta la edad, y es superior en las mujeres. Los principales padecimientos entre la población femenina son: hipertensión (40\%), diabetes (24\%) e hipercolesterolemia (20\%). Para los indicadores de salud mental: $17.6 \%$ presentó síntomas depresivos; $7.3 \%$, deterioro cognitivo, y $7.9 \%$, demencia. En el estado funcional: $26.9 \%$ reportó dificultad para realizar actividades básicas y $24.6 \%$ para actividades instrumentales (Manrique Espinoza, 2013, p. 323).

FIGURA 2. Causales de principales enfermedades crónico degenerativas en adultos en México

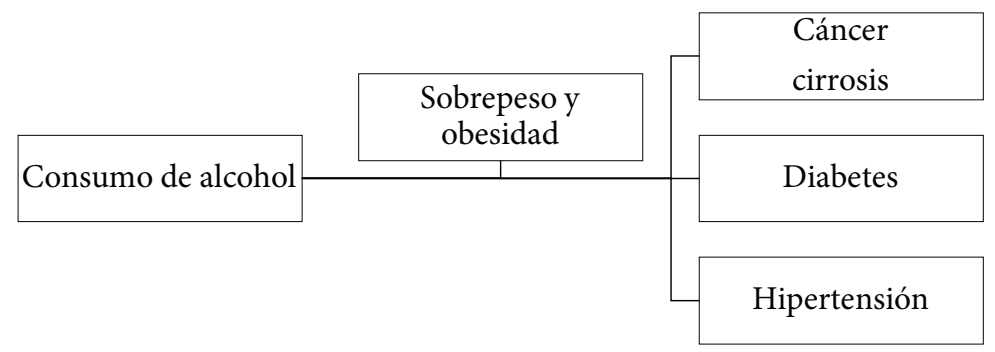

Fuente: Elaboración propia con base en el análisis de la sección.

Como se observa en el cuadro 3, tanto hombres como mujeres de la tercera edad padecen principalmente las mismas enfermedades crónico degenerativas.

CUADRO 3. Principales padecimientos de hombres y mujeres de la tercera edad

1. Hipertensión

2. Diabetes

3. Hipercolesterolemia

4. Depresión

Fuente: Elaboración propia con base en Manrique Espinoza (2013).

Si analizamos las causales de muerte en adultos mayores encontramos lo mismo, enfermedades crónico degenerativas en los primeros lugares. En el caso de hombres 
adultos mayores de 60 años, se encuentran en primer lugar las enfermedades isquémicas del corazón; en segundo, diabetes mellitus; en tercero, enfermedades cerebrovasculares, y en último lugar, las enfermedades pulmonares obstructivas. En el caso de mujeres, las causales son las mismas, pero en diferente orden. El primer lugar lo ocupa la diabetes mellitus; el segundo, las enfermedades isquémicas del corazón; el tercero, las enfermedades cerebrovasculares, y el último lugar, las enfermedades pulmonares.

CUADRO 4. Causales de muerte en adultos mayores

\begin{tabular}{ll}
\multicolumn{1}{c}{ Hombres } & \multicolumn{1}{c}{ Mujeres } \\
\hline 1. Enfermedades isquémicas del corazón & 1. Diabetes Mellitus \\
2. Diabetes mellitus & 2. Enfermedades isquémicas del corazón \\
3. Enfermedades cerebrovasculares & 3. Enfermedades cerebrovasculares \\
4. Enfermedades pulmonares & 4. Enfermedades pulmonares
\end{tabular}

Fuente: Elaboración propia con base en Conapo (2012).

Como podemos observar, actualmente la población adulta y adulta mayor, en México, muere por enfermedades crónico degenerativas, sobre todo en zonas urbanas. En zonas rurales, en las que vive un menor porcentaje de la población total, no se observan las mismas enfermedades ni causas de muerte. En 2010, en las zonas urbanas, las enfermedades crónicas no transmisibles contribuyeron con 71 $\%$ de la carga de enfermedad, a expensas del descenso de las causas transmisibles, maternas, de la nutrición y reproductivas (15\%) (Lozano et al., 2013, p. 583). Sin embargo, la tendencia es al descenso. Por ejemplo, las enfermedades con descensos significativos son las diarreicas (-90\%), las infecciones respiratorias (-65\%), las anomalías congénitas (-26\%), las complicaciones al nacer por prematurez (-55\%) y la tuberculosis, que pasó del lugar 18 al 26 (Lozano et al., 2013, p. 583). Así, la estructura epidemiológica del país es ligeramente diferente en las zonas rurales.

Es importante tener en cuenta que las enfermedades que ocasionan la muerte de una persona denotan en gran medida las condiciones socioeconómicas en las que esta nace, crece y se desarrolla. Los casos en que la población fallece por padecimientos infecciosos, nutricionales o relacionados con causas maternas, por lo general, indican niveles de desarrollo incipientes y condiciones de vida poco favorables para la salud, regularmente asociadas a las zonas rurales. Por ejemplo, acceso limitado a los servicios de salud y de educación, condiciones inadecuadas de vivienda (falta de drenaje y de energía eléctrica y hacinamiento), además de malos hábitos de higiene y conductas que favorecen la aparición de este tipo de enfermedades. En cambio, en las regiones donde es mayor el desarrollo socioeconómico la situación epidemiológica está dominada por enfermedades no transmisibles (crónico degenerativas) (Muradás, Solís \& Sánchez, 2014, p. 1). En materia de política 
pública, es importante tener presente este cuadro epidemiológico a la hora de realizar la asignación presupuestal, aspecto que analizaremos a detalle más adelante.

En síntesis, los principales problemas epidemiológicos que afectan la salud de la niñez en México son desnutrición, anemia, mortalidad infantil y obesidad. Problemas que se agudizan cuando se combinan con ciertas variables socioeconómicas y geográficas, de tal manera que es muy probable que un niño indígena que vive en una zona rural y es de estrato socioeconómico bajo padezca alguna de estas afectaciones a la salud. En el caso de los jóvenes, los principales problemas de salud están relacionados con la depresión, el consumo de alcohol y drogas, y se reflejan sobre todo en un elevado número de muertes por homicidios, accidentes de vehículo y suicidios. Estos problemas se agudizan entre los hombres. En el caso de adultos y adultos mayores de zonas urbanas, los principales problemas son enfermedades crónico degenerativas como diabetes mellitus, cirrosis, enfermedades isquémicas del corazón, enfermedades cerebrovasculares y cáncer. Prácticamente todas estas enfermedades están relacionadas con el consumo de alcohol y la obesidad. Además, es importante no dejar de lado que los trastornos mentales también están afectando a estos sectores de la población, sobre todo la depresión. En zonas rurales, el cuadro epidemiológico de los adultos es distinto; en ellas la población aún fallece por enfermedades diarreicas e infecciones respiratorias, lo cual denota las condiciones socioeconómicas en que viven.

A partir de la revisión que hemos ofrecido es posible esbozar el perfil epidemiológico de México. Es claro que las enfermedades con mayor incidencia en todos los grupos de población analizados se pueden englobar en unos cuantos grupos. Por consiguiente, como se ha dicho, la prevalencia de padecimientos crónico degenerativos es y seguirá siendo, al menos en el mediano plazo, la base de las causas de mortalidad en el país. Así, la estructura del gasto público en salud no puede limitarse a la atención paliativa de los padecimientos de este tipo, pues la presencia de obesidad y sobrepeso en los segmentos jóvenes de la población, así como el consumo de alcohol y tabaco, por ejemplo, delinea un panorama sombrío para los servicios de salud en México. Llanamente: si la asignación del gasto público en materia de salud sigue limitándose a la atención de las consecuencias de las enfermedades crónico degenerativas y no se diseñan e instrumentan grandes estrategias de prevención, eventualmente serán insuficientes los recursos para atender al cúmulo de pacientes nuevos que se añadirán a los ya diagnosticados. La lógica de la evolución de padecimientos como la obesidad, el sobrepeso o el consumo de alcohol es sencilla. Si estos individuos no cambian sus patrones de comportamiento, eventualmente se convertirán en pacientes con diagnóstico de diabetes, hipertensión, enfermedades cardio o cerebrovasculares, enfermedades hepáticas o cáncer. En el fondo de la discusión que hemos presentado en este apartado está el hecho de que el gobierno no ha modificado el paradigma de atención y aún no se comprende que los padecimientos crónico degenerativos constituyen, por su nivel de incidencia, una epidemia de proporcio- 
nes mayúsculas. En la idea de la epidemia radica el cambio de paradigma de política pública sanitaria que es urgente. Así como se redujo la incidencia de enfermedades transmisibles que antaño eran epidémicas en México, también pueden solucionarse los padecimientos no transmisibles con una política de salud preventiva.

\section{Proyecciones demográficas}

Al perfil epidemiológico general esbozado en el apartado anterior debe añadirse el segundo elemento del análisis causal que proponemos: las proyecciones demográficas. Un sistema de salud debe prepararse no solo para los problemas que enfrenta en el presente, sino también para los que enfrentará en el futuro. Por lo tanto, en materia de salud y decisiones públicas, es importante considerar la estructura poblacional de mediano y largo plazo de la sociedad que se esté atendiendo.

En México hay un proceso acelerado de envejecimiento que está transformando la estructura poblacional, incrementando la esperanza de vida y modificando el perfil epidemiológico de la población mexicana, de acuerdo con las estimaciones poblacionales. Los descensos en la fecundidad y la mortalidad han incidido sobre la magnitud y naturaleza de las causas de muerte, han sustituido los grupos más afectados y recorrido el momento de la muerte a edades cada vez más avanzadas.

De acuerdo con el último censo, en 2010 la población total de México era de 113.5 millones de habitantes ( $50.7 \%$ mujeres y $49.3 \%$ hombres), y la tasa de crecimiento fue de $1.5 \%$ anual entre 1990 y 2010. Por su parte, el segmento de población de adultos mayores registró 10 millones de individuos de 60 años o más, lo que representa el $9 \%$ del total de la población. Su tasa de crecimiento anual es superior a la de la población total con $3.8 \%$, lo cual implica que en 2020 habrá casi 16 millones de adultos mayores en el país. En la gráfica 1 se observa con mayor claridad cómo se invierte la pirámide poblacional. Como se puede ver, durante la década de los setenta y del dos mil la pirámide presenta una forma triangular en que predomina la base compuesta por jóvenes. En 2030 la pirámide adoptará una forma rectangular, en la que los adultos mayores comenzarán a ser la población más abundante. Finalmente, las proyecciones muestran que para 2050 los adultos mayores serán el grupo poblacional más grande por mucho.

En la actualidad hay más individuos mayores de 60 años que niños menores de cuatro, y las proyecciones indican que el envejecimiento demográfico es irreversible en el mediano plazo, pues persistirá durante varias generaciones. Este crecimiento también es una muestra del aumento en la esperanza de vida, que actualmente es de 76.4 años para la población en general. Sin embargo, la esperanza de vida saludable para la población en general es de 65.8 años, lo cual significa que los individuos viven 10.6 años con una carga de enfermedad y dependencia (Manrique Espinoza, 2013, p. 
323). Las causas de esos años de dependencia se encuentran en el perfil epidemiológico que hemos detallado. Cada vez más, las enfermedades crónico degenerativas han sustituido a las enfermedades transmisibles como causales de dependencia y muerte.

GRÁFICA 1. Pirámides demográficas México a través de los años

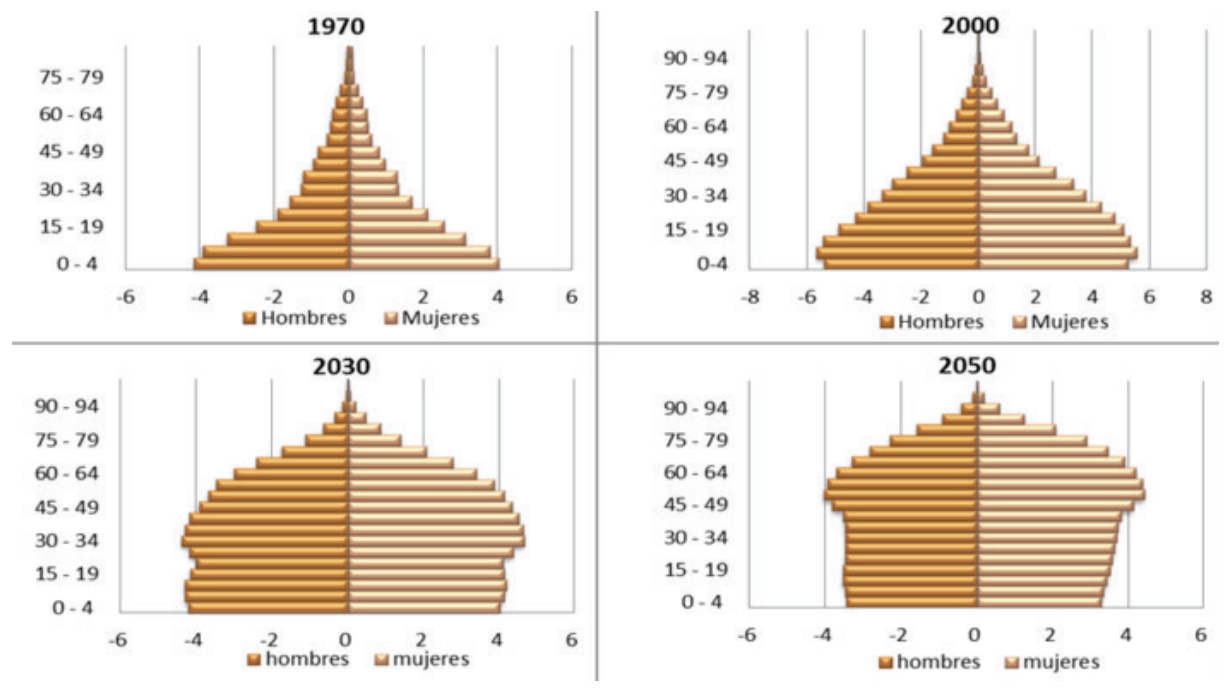

Fuente: Elaborado por el Centro de Investigación Económica y Presupuestal (CIEP) con información de CONAPO, proyecciones de población 2005 - 2050 e INEGI, censo de población de 1970 y censo de población de 2000.

Fuente: Maya (2012).

Si la población mexicana ahora vive más años y, en promedio, muere a edades mayores, este incremento en la esperanza de vida no necesariamente garantiza un buen estado de salud de las personas, pues el estudio de carga de la enfermedad de 2010 estimó que México presentaba una esperanza de vida saludable (sin discapacidad) de 67.1 años en las mujeres y de 63.6 años en los hombres, lo cual significa que ellas viven 11.3 años de su vida - casi siempre la última etapa-con discapacidad y los hombres aproximadamente 10 años (Lozano et al., 2013, p.583). Lo anterior implica una carga sustancial para el sistema de salud porque, en unos años, la mayor parte de la población será de adultos mayores viviendo más años con alguna enfermedad crónico degenerativa. En esta previsión se identifican dos problemas para el sistema de salud: la obligación de hacerse cargo de adultos mayores con padecimientos crónico degenerativos y la persistencia de esa obligación durante al menos un decenio. La creciente prevalencia de las enfermedades crónicas constituye un reto particular, pues compromete la funcionalidad, genera discapacidad, eventualmente conduce a la dependencia y representa, además, un incremento significativo en el costo de la atención a la salud. Se debe reconocer, 
por tanto, la importancia del estado funcional de los adultos mayores, ya que determina en gran medida la relación entre el envejecimiento poblacional y el gasto en salud (Manrique Espinoza, 2013, p. 324).

¿Los programas presupuestales como hipótesis casual?

Análisis de las asignaciones presupuestales de los ramos 12 (ss), 50 (IMSs) y 51 (ISSSTE) en relación con las casuales de los problemas epidemiológicos en México

En México, la inversión pública en salud ha pasado de $2.4 \%$ del producto interno bruto (PIB) en 2010 a $3.2 \%$ en 2016 (OCDE, 2016), y en los próximos años continuará incrementándose como consecuencia del perfil epidemiológico y poblacional que tendrá el país. En lo que respecta a los ramos del Presupuesto de Egresos de la Federación (PEF) que atienden la salud, analizamos los siguientes:²

CUADRO 5. Ramos analizados

\begin{tabular}{ll}
\multicolumn{2}{r}{ Ramo } \\
\hline 12 & Secretaría de Salud \\
50 & IMSS \\
51 & ISSSTE
\end{tabular}

En términos de presupuesto aprobado, el Ramo 12 del PEF, que corresponde a la Secretaría de Salud, pasó de 89.9 miles de millones de pesos en 2010 a 134.9 miles de millones en 2015, lo que representa un incremento en términos reales de 20 $\%{ }^{3}$ En el caso del Ramo 50 del PEF, que corresponde al IMSs, el presupuesto en este mismo periodo pasó de 320.3 miles de millones en 2010 a 497.6 miles de millones en 2015, para un incremento real de $24.28 \%$. En el caso del Issste, Ramo 51 del PEF, el presupuesto pasó de 107 miles de millones en 2010 a 208 miles de millones de pesos en 2015, para un incremento real del $55 \%$.

2 Es importante tener en cuenta que existen recursos asignados fuera de estos tres ramos que podrían también abordar indirectamente la problemática, sin ser su prioridad. Sin embargo, como se mencionó, se analizaron estos tres ramos porque son los únicos que tienen como principal prioridad velar por la salud pública nacional.

3 En los tres ramos (12, 50 y 51) los incrementos se calcularon con base en una inflación de enero 2010 a enero 2015 de 20.07 \% según INEGI. 
CARTA ECONÓMICA REGIONAL | ISSN 0187-7674 | AÑO 28 | NÚM. 118 | JULIO - DICIEMBRE 2016 | pp. 120-159

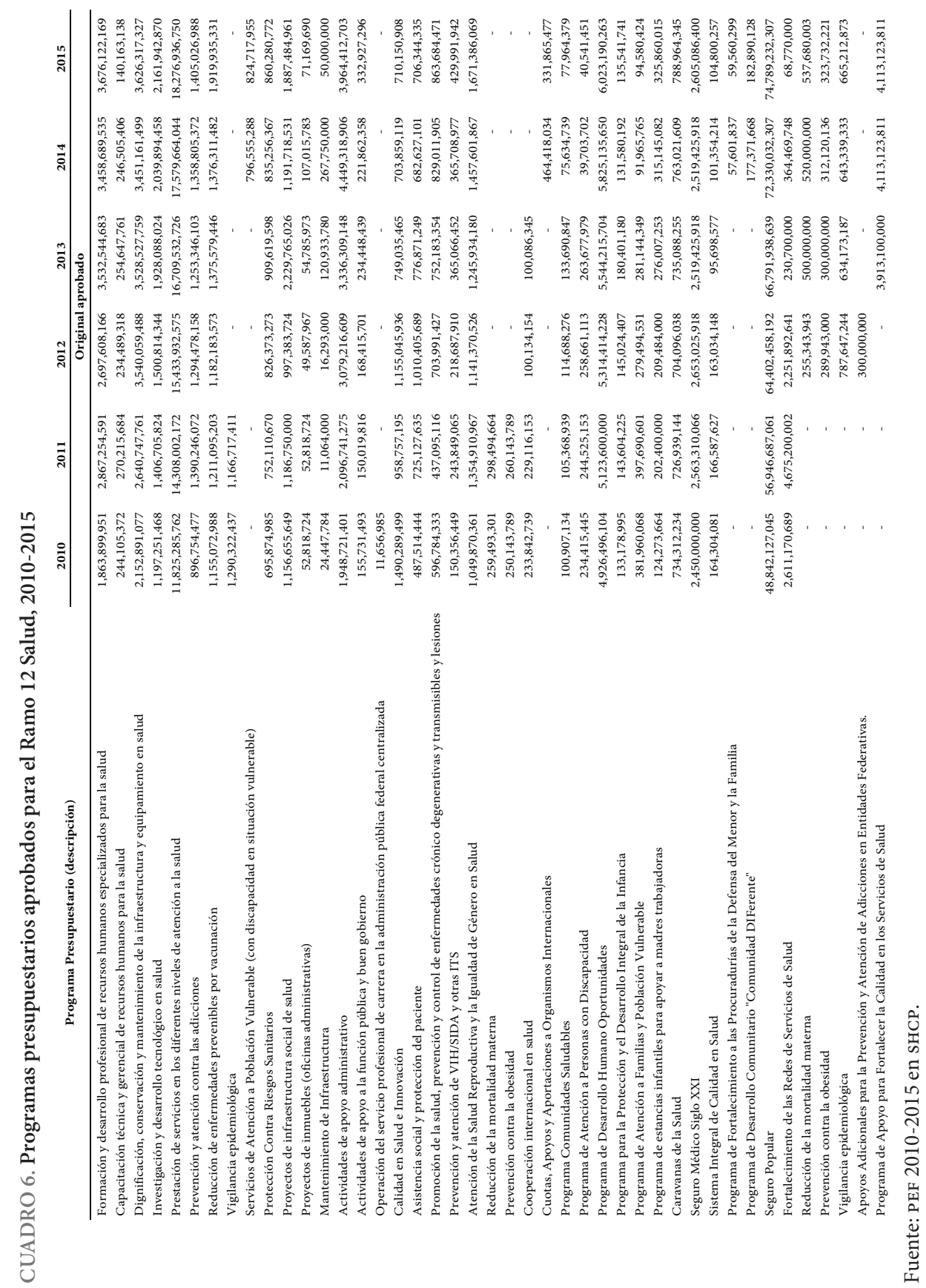


Anatomía de un problema público: la ausencia de la prevención de enfermedades crónico degenerativas... || G. TAPIA TÉLLEZ \& J. HERNÁNDEZ COLORADO

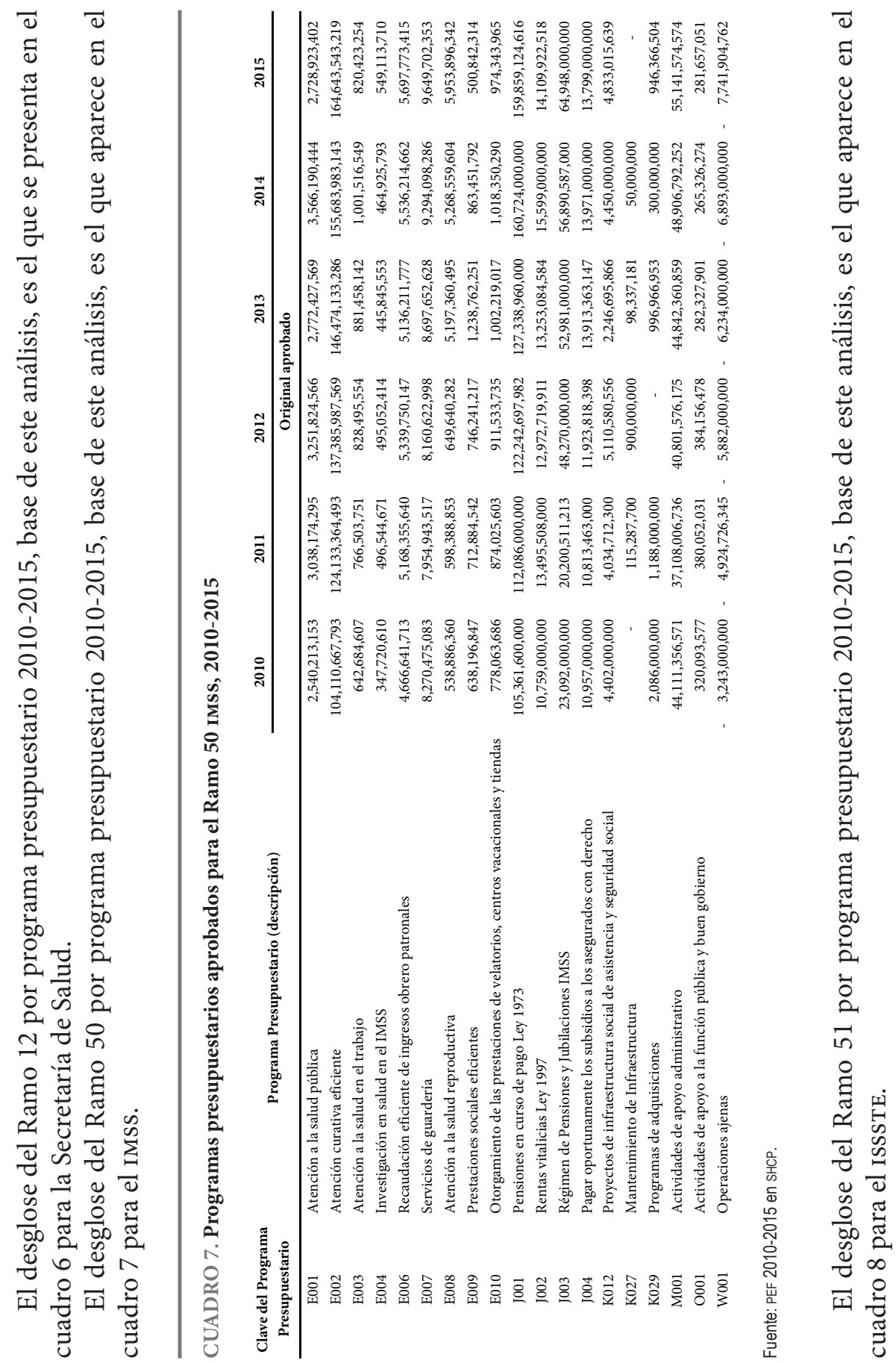


CARTA ECONÓMICA REGIONAL | ISSN 0187-7674 | AÑO 28 | NÚM. 118 | JULIO - DICIEMBRE 2016 | pp. 120-159

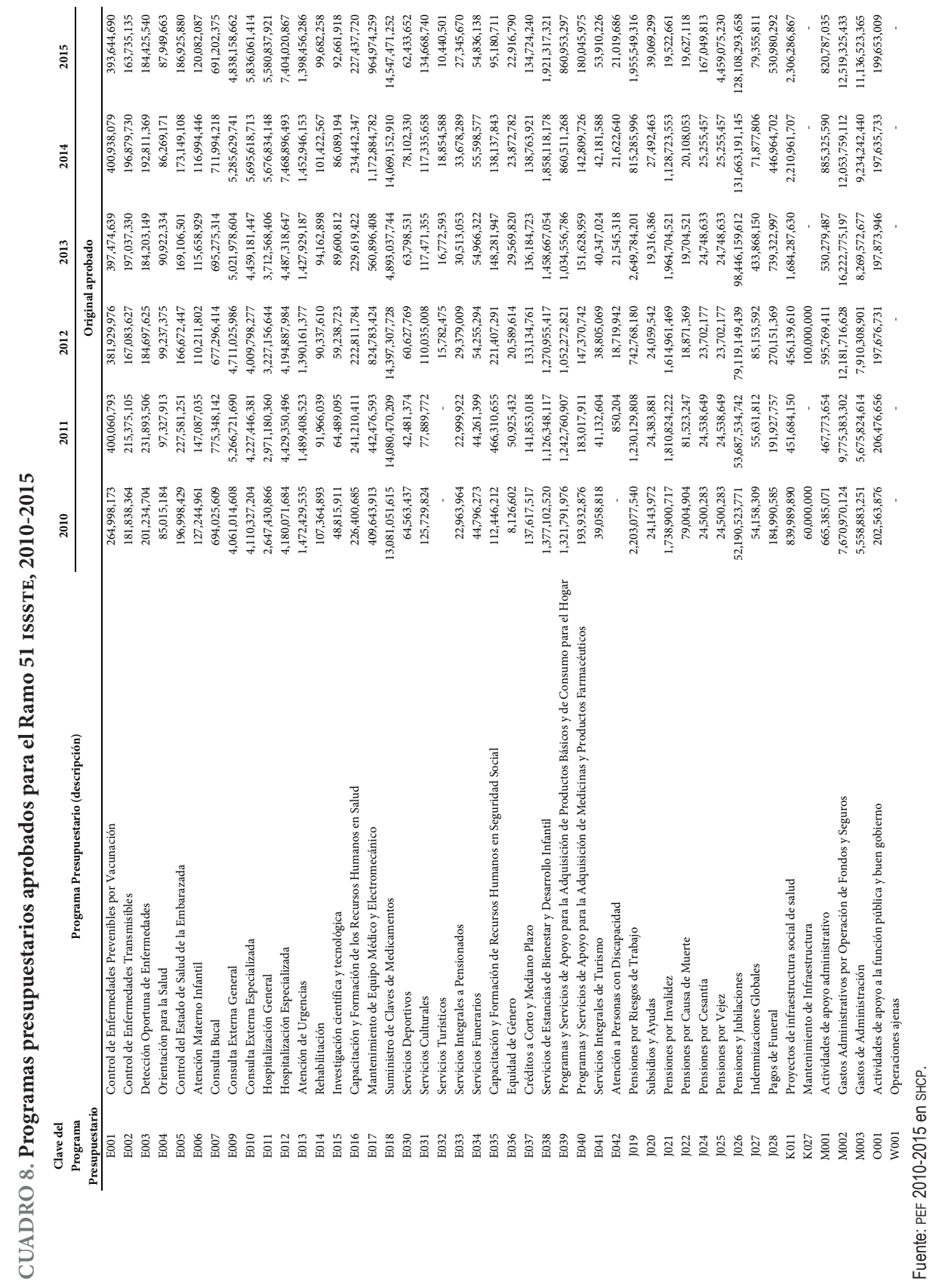


Aunque ha habido estos incrementos presupuestales, cuando se analizan los tres ramos por número de programas y por programas, se observa que prácticamente no han cambiado en los últimos cinco años. Como se ve en la gráfica 2, en el Ramo 12 (Secretaría de Salud), en 2010 se registraron 35 programas presupuestarios, mientras que en 2015 fueron 37. En el caso del Ramo 50 (IMss) se identificaron los mismos 18 programas en 2010 y 2015. En el IsssTe (Ramo 51) en 2010 había 43 programas y en 2015 se identificaron 44. Lo anterior, además de que refleja una inercia presupuestal en términos de programas en materia de salud en los tres ramos destinados a procurarla, también representa un riesgo para las finanzas públicas si se toman en cuenta las proyecciones demográficas, las tendencias epidemiológicas y los costos asociados a las mismas. ${ }^{4}$

GRÁFICA 2. Número de programas de ramos de salud, 2010 y 2015

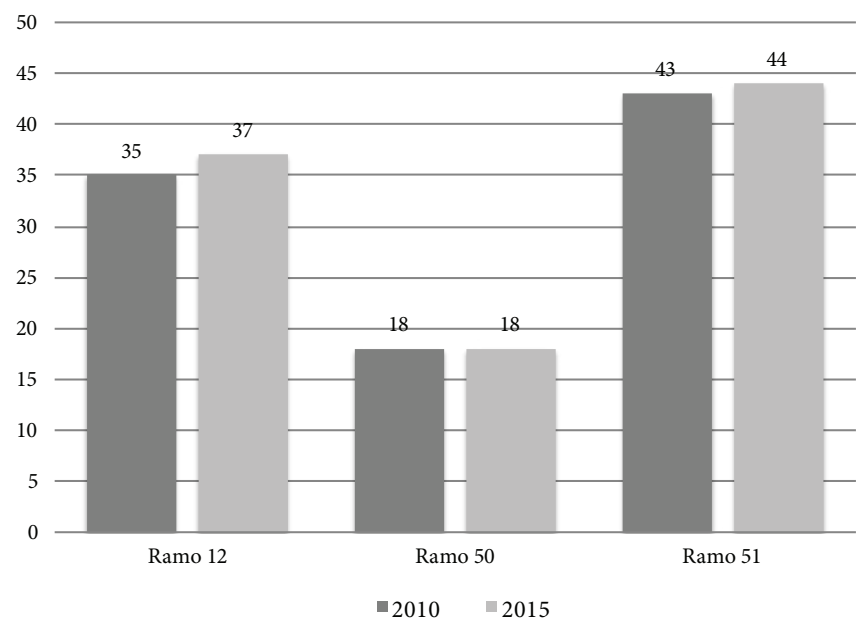

Fuente: Elaboración propia con base en información del PEF 2010-2015.

Como mencionamos al inicio, cuando la política pública se conceptualiza como respuesta o solución a problemas sociales, se convierte en una hipótesis causal, una acción - de características instrumentales-cuyos efectos empíricos producirían una situación social con atributos deseables (Aguilar, 2010, p. 32). En este sentido, una vez identificados los distintos problemas epidemiológicos y sus causales, se esperaría que el diseño presupuestal estuviera dirigido a su solución. O al menos a

$4 \quad$ La inercia presupuestal es uno de los elementos que dan cuenta de una tendencia incremental en el diseño de una política pública. Sin embargo, la perspectiva incremental en una política funciona en países donde las cosas "van bien" y las políticas públicas están dando buenos resultados, pero no en aquellos donde la bola de nieve se va haciendo más grande (Dror), como ocurre en México con los problemas epidemiológicos. 
dos ámbitos de acción que, en el futuro, podrían prefigura una solución: la atención a la población ya diagnosticada y la prevención para tratar de detener el incremento de enfermos crónico degenerativos. El énfasis en la prevención, además de ser deseable para la política pública de salud, dado el perfil epidemiológico del país, está consagrado en el artículo 2 de la Ley General de Salud, donde se detalla el derecho a la protección social en este rubro. Asimismo, en su artículo 27, caracteriza los servicios básicos para la materialización del derecho a la protección social en salud, entre los cuales se encuentra la prevención de enfermedades de atención prioritaria, transmisibles y accidentes. En todo caso, nuestro análisis también remite a la necesidad de definir con claridad todos los padecimientos del perfil epidemiológico actual del país como de atención prioritaria.

Las preguntas guía de esta investigación, que analiza los ramos 12,50 y 51 entre 2010 y 2015, son las siguientes: ¿cuál es la relación entre estos programas presupuestales y los principales padecimientos y sus causas en los distintos estratos de edad de la población?, ¿qué se puede decir en relación con las brechas de desigualdad que se observan por región geográfica?, ¿qué se puede decir en relación con las enfermedades por nivel socioeconómico?, ¿con qué criterios y de qué manera se utiliza el gasto destinado a la prevención de los principales padecimientos?

Comencemos por analizar los problemas epidemiológicos en los niños y las acciones presupuestales de los programas que los atienden. Como identificamos, en el grupo de población niños uno de los principales problemas es la inseguridad alimentaria, que se asocia con desnutrición, anemia, talla y obesidad. Hemos dicho que estos problemas se acentúan en niveles socioeconómicos bajos y en la población indígena. En el Ramo 12, correspondiente a la Secretaría de Salud, hay solo un programa que, entre sus muchos otros objetivos, asigna un espacio al problema de la desnutrición en los sectores menos favorecidos, el programa Prospera, ${ }^{5}$ el cual consiste en acciones de salud dirigidas a la comunidad y suplementos alimenticios. A Prospera le corresponde 4.47 \% del total del presupuesto del Ramo 12 (2015). Otra parte de la asignación presupuestal que también beneficia a la niñez, aunque no se dirige a las causales de los principales problemas epidemiológicos que enfrentan actualmente, es el de vacunación. Para este propósito, en el Ramo 12 hay un programa cuya asignación representa el $1.42 \%$ del presupuesto total del ramo en 2015.

Además de Prospera y el programa de vacunación, se identificaron tres programas dirigidos específicamente a los niños; sin embargo, tienen que ver más con el derecho al acceso a la salud y los derechos humanos que con las principales causales de los problemas epidemiológicos que se presentan en este segmento de edad. Por ejemplo, se identificaron el Programa para la Protección y el Desarrollo Inte-

Hay que señalar que este programa también es operado y ejerce recursos en otros ramos del presupuesto federal, como el de desarrollo social o educación. 
gral de la Infancia, ${ }^{6}$ el Programa de Fortalecimiento a las Procuradurías de Defensa del Menor y la Familia, y el Seguro Médico Siglo xxi, que es una especie de Seguro Popular para niños con fines sobre todo curativos.

En el Ramo 50, que corresponde al IMss, hay solo un programa dirigido a este grupo etario, el de Servicios de Guardería, que representa el $1.94 \%$ de los recursos aprobados. Sin embargo, como sucede en el Ramo 12, el programa no está dirigido a la solución de los problemas epidemiológicos específicos que enfrenta la población de esta edad. En el caso del Ramo 51, que corresponde al Issste, se identifican tres programas dirigidos a la niñez; pero, nuevamente, no tienen como objetivo solucionar los problemas epidemiológicos que padecen. Son el Programa de Control de Enfermedades Prevenibles por Vacunación, el Programa de Atención Materno Infantil y el de Servicios de Estancias de Bienestar y Desarrollo Infantil. En este ramo, además, se identificó un programa dirigido a la cuestión de la inseguridad alimentaria, el Programa y Servicio de Apoyo para la Adquisición de Productos Básicos y de Consumo para el Hogar. Cabe aclarar que el programa es para las familias en general y no solo para la niñez. Estos cuatro programas representan el $1.58 \% \mathrm{del}$ presupuesto del ramo.

Igual que sucede con el resto de los grupos poblacionales, en esta edad no se observa en la Secretaría de Salud, el IMSS y el ISssTe un énfasis en la prevención vía programas nutricionales para solucionar los problemas de inseguridad alimentaria, desnutrición y obesidad que se observan sobre todo en los niveles socioeconómicos bajos. El Seguro Popular, por ejemplo, que forma parte del Ramo 12, y entre sus cientos de espacios de cobertura incluye uno para acciones preventivas en la niñez por grupos quinquenales; sin embargo, se desconocen las acciones específicas en este sentido. Las cifras que hemos mencionado evidencian la incapacidad que ha habido hasta el momento para diseñar una estructura de gasto que atienda las principales causas de los problemas epidemiológicos del grupo de población correspondiente a niños. Cuando se identifica un problema público transversal y las acciones de política pública se dirigen a atender de manera muy general los distintos ámbitos del mismo con frecuencia sucede lo que se puede observar en los ramos 12, 50 y 51 del PEF: la capacidad del gasto federal para atender las causales de los problemas epidemiológicos de la infancia se diluye entre varias líneas de acción que no necesariamente las atienden.

La población indígena, ${ }^{7}$ por su parte, que como mencionamos padece en mayor medida problemas de inseguridad alimentaria, desnutrición y talla, no se ve

\footnotetext{
6 Este programa está a cargo del Sistema Nacional para el Desarrollo Integral de la Familia. Sus recursos se ejercen por medio de los sistemas estatales.

7 Según datos del Censo General de Población y Vivienda 2010, había en México 6.6 millones de indígenas, consideradas así por ser hablantes de una lengua originaria. De ellos, el $61 \%$ residía en poblaciones de menos de 2,500 habitantes.
} 
favorecida por una mayor cantidad de programas o más presupuesto en materia de salud. En el Ramo 12 no se identificó ningún programa para atender a esta población. Existen, por ejemplo, programas como el de Caravanas de la Salud, que benefician a la población indígena, ya que se dirigen a los sectores poblacionales con "más hambre"; sin embargo, el énfasis no está en la niñez indígena. ${ }^{8}$ En los programas que opera la Comisión Nacional para el Desarrollo de los Pueblos Indígenas (CDI), que está sectorizada en la Secretaría de Hacienda y Crédito Público, existen líneas de acción que podrían atender las desventajas de la atención integral a la niñez indígena, pero su énfasis es general y no específico. En los ramos 50 y 51 no existen programas dirigidos específicamente a este grupo poblacional en relación con la nutrición, aunque los programas de Liconsa, Diconsa y el Programa de Apoyo Alimentario (PAL) de la Secretaría de Desarrollo Social (Sedesol) probablemente los beneficien porque se dirigen a la población con menos recursos, que en México coincide que es también la población indígena. ${ }^{9}$

En el caso de los jóvenes, como hemos señalado, las principales causas de muerte son los homicidios, accidentes automovilísticos y suicidios, todas relacionadas con el consumo de alcohol y otras drogas. En el caso de los suicidios, muchos están relacionados con unas condiciones tales como la depresión. El análisis de los programas presupuestarios de los ramos 12, 50 y 51 deja ver la existencia de un descuido de este sector de la población, pues no hay un solo programa dirigido específicamente a los jóvenes. En 2012, en el Ramo 12 había dos programas dirigidos a las adicciones; no obstante, en la actualidad solo hay uno encaminado a atender este problema de manera general, no exclusivamente dirigido a los jóvenes. El gasto en este programa representó el $1.04 \%$ del total del Ramo 12 en 2015. ${ }^{10}$

8 Para completar este análisis, sin embargo, tendría que analizarse el Ramo 6 del PEF, ya que en la SHCP existen decenas de programas dirigidos a población indígena. Habría que analizar cuántos y cuáles de ellos están relacionados con el tema de la salud.

9 Es importante tener en cuenta que en otros ramos, como el 20, que corresponde a la Sedesol, se identificaron " 5 " programas relacionados con aspectos de nutrición, algunos de ellos dirigidos especialmente a la niñez, tales como el Programa de Adquisición de Leche Nacional y el Programa de Abasto Social de Leche, a cargo de Liconsa, y el Programa de Abasto Rural de Diconsa. Además, entre los programas no dirigidos específicamente a este grupo etario pero sí al tema de nutrición se identificó el Programa de Apoyo Alimentario y los Comedores Comunitarios. Estos cinco programas representan el $11.77 \%$ del presupuesto total de Sedesol en 2015. Finalmente, otros programas de Sedesol dirigidos a la niñez en particular, aunque no necesariamente relacionados con las causales epidemiológicas analizadas en esta edad, se encuentra el Programa de Estancias Infantiles para apoyar a madres trabajadoras, que se lleva el 3.33 \% del presupuesto en 2015. En el Ramo 11, que corresponde a la Secretaría de Educación Pública (SEP), existen siete programas relacionados con el deporte, infraestructura para el deporte y cultura física que sí están relacionados con este tipo de problemáticas, su presupuesto corresponde al $1.25 \%$ del total de presupuesto 2015 de la SEP.

10 No debemos dejar de lado, sin embargo, que entre los cientos de esquemas de cobertura del Seguro Popular existe una Consejería de Adicciones en Adolescentes. Pero su utilidad y funcionamiento se desconocen, además de que el Seguro Popular atiende solo a un sector de la población joven. 
Además de estos temas, en los jóvenes se identificaron problemas de obesidad importantes; sin embargo, como veremos a continuación, en los ramos analizados la prevención no pareciera ser una prioridad. El Seguro Popular, como sucede con el grupo de niños, tiene en su esquema de cobertura Acciones Preventivas para Jóvenes pero se desconoce cuáles son estas acciones, su impacto y qué porcentaje representan del presupuesto destinado al Seguro Popular. ${ }^{11}$ En el IMss y el Issste, como mencionamos, no hay un solo programa dirigido a jóvenes en específico, y tampoco para tratar problemas de obesidad. En materia de prevención en el IsssTE (Ramo 51) existe solo el programa de detección oportuna de enfermedades; sin embargo, no está dirigido específicamente a este grupo etario y su presupuesto es del $0.09 \%$ del total de este ramo. ${ }^{12}$

Con respecto a los adultos, el problema de las enfermedades crónico degenerativas afecta principalmente a hombres y mujeres que viven en zonas urbanas. Estas enfermedades son: diabetes, problemas cardiacos y cerebrovasculares, cirrosis y cáncer de mama, en el caso de las mujeres. De los 37 programas que componen el Ramo 12 en 2015, solamente uno tiene como propósito específico atender de manera preventiva estos problemas y significa tan solo el $0.64 \%$ del presupuesto 2015 del ramo. En relación con esto, por ejemplo, el gasto en causales como obesidad, tan importantes en estas enfermedades, representa únicamente el $0.30 \%$ vía dos programas, el de Comunidades Saludables y Prevención contra la Obesidad. En el Ramo 50, que corresponde al IMss, no se identifica tampoco ningún programa dirigido a la prevención y en el ISSSTE, como mencionamos, hay solo uno de prevención, el de Detección Oportuna de Enfermedades, cuyo presupuesto es mínimo. En ninguno de estos casos se hace mención alguna en materia de obesidad. Los datos anteriores evidencian falta de atención al tratamiento; y no se diga a la prevención de enfermedades crónico degenerativas que, como hemos dicho en el apartado anterior, son una suerte de espada de Damocles para el sistema de salud mexicano. En la información que se reseña en este párrafo, además, es diáfano que la atención preventiva de ese tipo de padecimientos es mínima. Y surge el cuestionamiento acerca de qué es más grave —en perspectiva de futuro-, la nula prevención de tales enfermedades o el hecho de que las acciones preventivas reciban atención exigua.

$11 \quad$ El acceso al presupuesto desagregado del Seguro Popular resulta complicado. Este tema incluso ha sido durante varios años objeto de demandas por parte de grupos de la sociedad civil en pos de transparencia presupuestaria.

12 En otros ramos, como el Ramo 11, que corresponde a la SEP, se identificaron tres programas dirigidos específicamente a los jóvenes. El primero de ellos, directamente relacionado con estos problemas, es el de reinserción académica de los jóvenes integrantes de bandas y pandillas. El segundo, dirigido también a un problema importante para las mujeres en esta etapa, es el de becas de apoyo a la educación básica de madres jóvenes y jóvenes embarazadas. El tercero es el subsidio a programas para jóvenes, presente también en el Ramo 20 de Sedesol, que atiende transversalmente varios de los problemas que enfrenta la juventud actualmente mediante apoyos para emprendimiento, vivienda, salud y educación. 
El consumo de alcohol es causa de muchas enfermedades crónico degenerativas, pero en los ramos 12, 50 y 51 la prevención de esta problemática ni de lejos ocupa un lugar prioritario. En el Ramo 12, como mencionamos, solo existe un programa dirigido al problema de las adicciones y se le destina el $1.04 \%$ del gasto total del ramo. En lugar de incrementar el número de programas para atender esta causal, que abarca gran parte de los problemas epidemiológicos del país, en 2013 se decidió disminuir el presupuesto del Ramo 12 y quitar uno de los dos programas que existían. Lo anterior es evidencia de que el aspecto preventivo no es una prioridad - por lo menos no se refleja en el gasto del ramo que nos ocupa- y, en segundo lugar, no se observa una hipótesis causal entre los principales problemas epidemiológicos, sus orígenes y la asignación presupuestal. En el Ramo 50 del IMSs y el Ramo 51 del ISssTe tampoco se identificó ningún programa para combatir adicciones o reducir el consumo de alcohol entre la población. Es decir, hasta el momento el diseño presupuestal ha ignorado la urgencia de vincular los problemas epidemiológicos actuales y futuros con los comportamientos y las causas que es posible atajar con acciones preventivas. Incluso por lógica económica, es urgente que la planeación presupuestal en materia de salud ponga atención en la prevención pues, de seguir en la trayectoria actual, los problemas epidemiológicos que hemos mencionado alcanzarán niveles casi inmanejables en términos de política pública. Y esta predicción no es resultado de proyecciones sociológicas, sino de investigaciones y datos de la ciencia médica mexicana.

Un estudio reciente del Instituto Nacional de Salud Pública en el que se estima la carga atribuible a la exposición a diversos factores de riesgo que son determinantes en la aparición de numerosos problemas de salud encontró que, en el caso de los hombres, destacan el consumo de alcohol, el sobrepeso, la obesidad, los niveles elevados de glucosa en sangre, la presión arterial alta y el consumo de tabaco como los factores de riesgo más importantes y responsables de $35.6 \%$ de los años de vida saludable perdidos en 2010. En el caso de las mujeres, los factores que explican las principales enfermedades crónico degenerativas tienen que ver con sobrepeso, obesidad, glucosa elevada, hipertensión, sedentarismo y consumo de alcohol y tabaco, a las que en conjunto se responsabiliza del $40 \%$ de la carga de los años de vida saludable perdidos (Lozano et al., 2013, p. 584). En ambos casos el sobrepeso y la obesidad son factores explicativos importantes en materia de prevención. Sin embargo, en los ramos 12, 50 y 51, que son los destinados a la salud, no se identificó ningún programa cuyo objetivo sea atender este problema. Contrario a lo que se esperaría, el único programa que existía en el Ramo 12 entre 2010 y 2011, denominado Prevención contra la Obesidad, desapareció en 2013 y actualmente no se asignan recursos de ninguno de estos ramos a su prevención. Y lo mismo sucede con el tema de la actividad física.

Gran parte de las enfermedades crónico degenerativas y causales de muerte en todas las edades son prevenibles, lo cual podría ser la esperanza a futuro en términos de sostenimiento del sistema de salud. Los elevados costos curativos de estas enferme- 
dades y la transición demográfica, que nos garantiza un incremento significativo de la población adulta mayor que será afectada por este tipo de padecimientos, podrían poner en riesgo la viabilidad del sistema de salud y de las finanzas públicas mexicanas en su conjunto, pues será muy complicado atender con los recursos disponibles a los sujetos que padezcan estas enfermedades. Es claro que se requiere reorientar el gasto público en salud para prevenirlas y evitar que alcancen el un futuro las magnitudes inmanejables que se prevén. Esta dinámica sería difícil de controlar y alteraría -si se manifiesta - el funcionamiento global de las finanzas públicas del país.

Respecto a la brecha en salud entre las zonas urbanas y las rurales, identificamos en el Ramo 12 dos programas que la atienden: el de Caravanas de Salud, cuya porcentaje del presupuesto total es del 0.58 del total del Ramo 12, y Prospera que, como ya mencionamos, representa $4.47 \%$. De suerte que el vínculo causal que sugerimos establecer ya existe en este caso. Sin embargo, el análisis de los ramos 50 y 51 no refleja este objetivo vía el gasto, es decir, no se observa todavía ningún programa con asignación presupuestal cuyo objetivo sea atender esta problemática.

Otro aspecto que se ha dejado de lado y que afecta a los grupos poblacionales jóvenes, adultos y adultos mayores, es el de la salud mental. En el análisis de los 37 programas presupuestarios del Ramo 12 no se encontró uno solo dirigido a esta problemática en específico. El Seguro Popular tiene en su esquema de cobertura acciones preventivas como la detección temprana de trastornos alimentarios, depresivos, de ansiedad y adicciones; estas acciones están distribuidas por grupos etarios. Sin embargo, se desconoce cuáles son estas acciones, el impacto que tienen y qué porcentaje representan del presupuesto destinado al Seguro Popular, pues la glosa a este nivel de detalle no existe en el presupuesto. ${ }^{13}$ En el análisis de los ramos 50 y 51 tampoco se observó ningún programa dirigido a atender trastornos mentales.

Por otra parte, un aspecto positivo que se identificó en el análisis es el gasto que se dirige a adultos mayores en materia preventiva. A diferencia de lo que sucede con otros grupos poblacionales, en el caso de los adultos mayores el Ramo 12 sí destina recursos a la promoción del envejecimiento sano y activo vía Seguro Popular y el Catálogo Universal de Servicios de Salud (Causes), a través de la inversión en prevención y promoción de la salud y manteniendo una perspectiva de curso de vida. De acuerdo con una investigación reciente (Manrique Espinoza, Salinas Rodríguez, Moreno Tamayo, Acosta Castillo \& Sosa Ortiz, 2013) actualmente, dentro del Causes se incluyen casi todas las acciones preventivas necesarias para este fin. Recordemos que el Seguro Popular se lleva el 55.4 \% del presupuesto del Ramo

13 Dada la cantidad de acciones incluidas en el Seguro Popular y el porcentaje de los recursos que se asignan a este programa en el Ramo 12, sería necesario, para completar este análisis, tener acceso a la manera en que el Seguro Popular distribuye los recursos que se le asignan entre las distintas acciones que realiza, así como conocer las evaluaciones, si es que existen, de sus resultados. Desafortunadamente, este es un vacío importante aún en materia de transparencia presupuestaria. 
12. En los ramos 50 y 51, desafortunadamente, no se identificaron programas específicos para atender los problemas epidemiológicos de este grupo de población. Aunque existen programas para adultos mayores, se centran en cuestiones como el régimen de pensiones y jubilaciones en el caso del Ramo 50 (IMss) y servicios funerarios, pensiones por vejez, pagos de funeral y pensiones y jubilaciones en el caso del Ramo 51 (ISSSTE).

Para concluir, es importante hacer un último apunte, el cual abunda en que el aspecto preventivo y las causas de los principales problemas epidemiológicos del país no son una prioridad en materia presupuestal vía los ramos 12, 50 y 51 . Para ello segmentamos el gasto del Ramo 12 por temas y observamos que $17 \%$ del presupuesto total del mismo se gasta en cuestiones relacionadas con capacitación de recursos humanos, infraestructura y administración; un $18 \%$ se destina a mejorar la calidad de los servicios de salud que se ofrecen. Si a ello le sumamos el $55.4 \%$ del presupuesto que se asigna a Seguro Popular, encontramos que a estos tres aspectos se destina el $90.44 \%$ del presupuesto del Ramo 12. El gasto en prevención, como se puede observar en la gráfica 3 , es insignificante.

GRÁFICA 3. Presupuesto 2015 para el Ramo 12 por temas
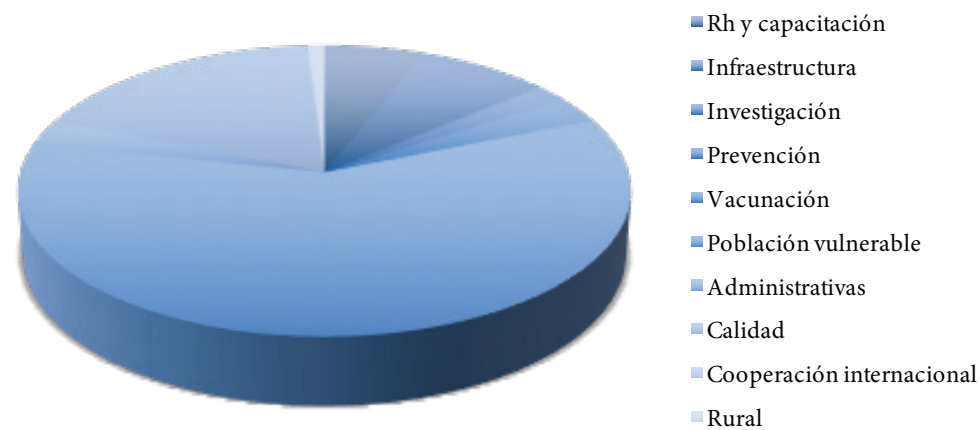

Fuente: Elaboración propia con base en el Ramo 12 del PEF PEF 2015 aprobado.

En el Ramo 50 la situación es similar ya que, como mencionamos, no existe ningún programa presupuestal que tenga como objetivo la prevención o contención de los problemas epidemiológicos que aquejan a la población en México. Como se puede observar en la gráfica 4 , gran parte del presupuesto se destina a cuatro programas: atención curativa eficiente, pensiones en curso de pago Ley 1973, pensiones y jubilaciones y actividades de apoyo administrativo. Ninguno de ellos tiene como objetivo la prevención. 
GRÁFICA 4. Principales programas y su asignación presupuestal 2015, Ramo 50: IMss

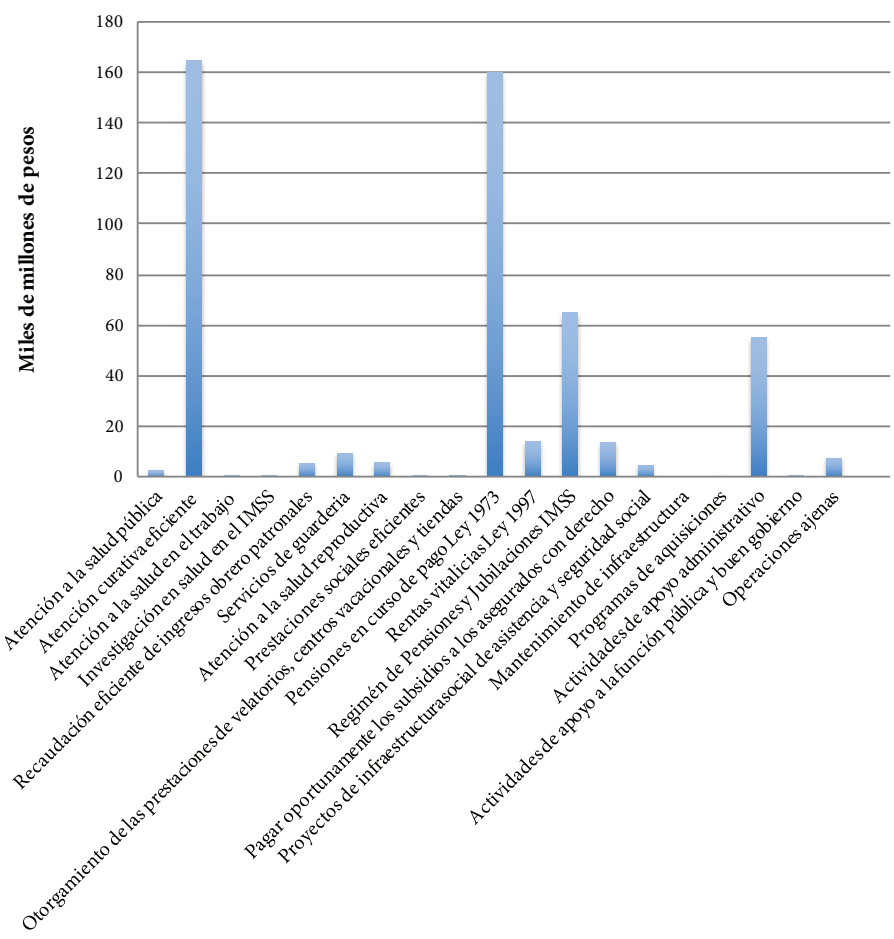

Fuente: Elaboración propia con base en el Ramo 50 del PEF 2015 aprobado.

En el Ramo 51, correspondiente al IsssTE, solo se identifica un programa dirigido a la prevención, el de detección oportuna de enfermedades. Sin embargo, como mencionamos, se le asigna el $0.09 \%$ del total del presupuesto de este ramo. $\mathrm{Al}$ igual que en el Ramo 50, una parte sustancial del presupuesto del IMss se destina a pago de pensiones y jubilaciones y prácticamente nada del mismo a programas dirigidos a la prevención.

El análisis de los tres ramos deja ver la poca importancia que se da a las tareas de prevención de las principales enfermedades crónico degenerativas, dados los programas y sus asignaciones. El análisis de los ramos pareciera indicar que el gasto del gobierno federal en materia de salud no tiene como prioridad la atención de los principales problemas epidemiológicos actuales y sus causales. El análisis epidemiológico muestra que el aspecto preventivo debería ser actualmente prioritario en cuestiones como alcoholismo, nutrición y obesidad. La evidencia señala que el gasto es muy bajo en estos rubros. 
GRÁFICA 5. Principales programas y su asignación presupuestal 2015, Ramo 51: ISssTE

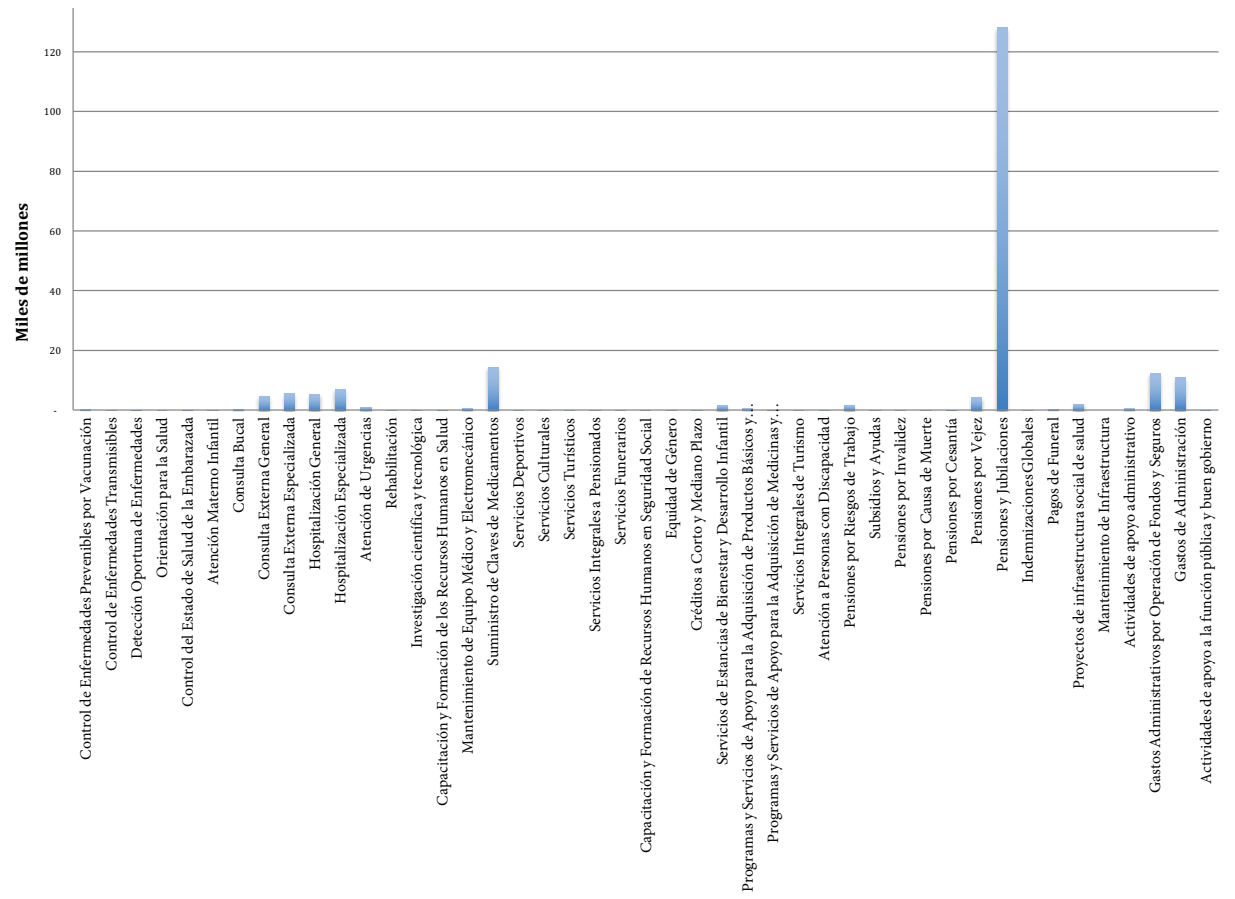

Fuente: Elaboración propia con base en el Ramo 51 del PEF 2015 aprobado.

Propuestas de reconfiguración del gasto en salud vía política pública

La relación causal que se planteó al inicio - y que hemos intentado demostrar con los argumentos presentados hasta ahora - sirve a la investigación en materia de política pública, pues deja claro que las prioridades del gasto en materia de salud no están enfocadas en la prevención de las enfermedades. No se debe perder de vista que el gasto en acciones preventivas, como lo hemos dicho, empata con la lógica de disminución del gasto futuro y puede considerarse gasto de inversión si se tiene en cuenta que el objetivo de largo plazo es evitar el aumento desproporcionado de la prevalencia de los principales padecimientos crónico degenerativos que hemos mencionado y que son, a la vez, las principales causas de muerte en el país, y lo serán en el futuro.

Otro de los fines de este artículo, además de analizar la situación epidemiológica y el diseño presupuestal de los ramo 12, 50 y 51 para dejar claro el divorcio entre las prioridades de los ramos del PeF destinados a la salud y la realidad epidemiológica del país, es proponer, a partir de estos dos análisis, algunos elementos 
para el diseño y la corrección de la política nacional de salud. La parte nodal de la propuesta se centra en el aspecto preventivo.

\section{Cuatro líneas generales de acción}

Como base para el viraje que, según la evidencia, es necesario en la planeación del gasto sanitario en México, se requiere tomar en cuenta las distribuciones poblacionales y las causales relacionadas con estas. Es decir, es necesario que en la planeación se observen políticas diferenciadas con base en las características geográficas de la enfermedad, las condiciones de vulnerabilidad de los que las padecen, el sexo y la edad. El análisis de los ramos presupuestarios deja ver un vacío importante en este sentido.

Una segunda línea de acción general tiene que ver con los incrementos del gasto público en salud. Es importante que tales incrementos, que de suyo no son cuestionables, por la materia a la que se dirigen, se reflejen en políticas que no solo se encaminen a servicios curativos, infraestructura o gasto administrativo, sino que permitan la modificación del énfasis de la política de salud hacia la prevención, único aspecto que contribuye a hacer sostenible el sistema a futuro. Es posible entender la inercia con que se ha manejado el diseño presupuestal en materia de salud en México, pues las prioridades han pasado varias décadas inalteradas y la dinámica sanitaria del país sigue exigiendo recursos en acciones curativas; sin embargo, las investigaciones médicas - a diferencia de los criterios valorativos de los diseñadores del presupuesto - prefiguran un panorama oscuro para la sostenibilidad del sistema sanitario. Gran parte de las enfermedades crónico degenerativas y causales de muerte son prevenibles. Asimilar el gasto en prevención como gasto de inversión es acaso la mejor forma de comprender que de la planeación preventiva que se haga ahora dependerá el perfil epidemiológico de las próximas décadas. Sin embargo, como pudimos ver y lo han identificado organismos internacionales, el gasto en administración (burocracia) de las instituciones de salud en México es uno de los más altos de la Organización para la Cooperación y el Desarrollo Económicos (oCDE, 2015).

Asociada al gasto y la cantidad de recursos que se asignan al Seguro Popular cada año (55.4\%), es necesaria también una mayor transparencia presupuestaria que permita tener acceso a los mecanismos por los cuales se distribuyen los recursos que se le asignan. Con esta información disponible es posible proponer nuevas aristas de política pública y elaborar explicaciones sobre el énfasis que está poniendo el gobierno federal, por medio de los ramos destinados a la salud: 12, 50 y 51, en materia de prevención epidemiológica. Asimismo, es posible evaluar la eficacia que está teniendo cada línea de acción actual.

Como tercera línea transversal de acción, las instituciones federales de salud podrían diseñar un Plan Nacional de Prevención de las Enfermedades Crónico 
Degenerativas ${ }^{14}$ en el que participen los tres niveles de gobierno y que esté dirigido a privilegiar la prevención. Este plan debe tener objetivos de mediano y largo plazo y su principal propósito ha de ser modificar los hábitos que están en el origen de estas enfermedades. La articulación de un esquema de coordinación en materia de prevención en salud, por el paradigma actual - que se limita a la curación-, tiene que partir necesariamente de un cambio en la idea de la gestión sanitaria en el país y, por supuesto, de la asimilación de que la circunstancia epidemiológica, que hemos descrito como una espada de Damocles, puede convertirse en motivo de emergencia nacional tanto en el ámbito médico como en el de las finanzas públicas.

La cuarta línea general de acción tiene que ver con el establecimiento de redes de colaboración entre universidades, centros de investigación y decisores públicos de los ámbitos federal, estatal y municipal, con la finalidad de trabajar en el diseño de las políticas públicas focalizadas que permitan atender las particularidades epidemiológicas que se presentan en el territorio nacional. Estas condiciones varían según la región, de manera que sería poco útil pensar en criterios de prevención y atención unívocos que pretendan solucionar los problemas descritos como si el país fuera un todo unificado y homogéneo. Así, es necesaria la coordinación por niveles de gobierno tanto en la planeación con objetivos preventivos y con base en características locales específicas como en la inclusión de actores relevantes que conocen los aspectos científicos del problema y han alertado, desde hace varios años, de los peligros que entraña a futuro el perfil epidemiológico de México.

Si cabe, como una línea transversal adicional, es preciso tener en cuenta que la sujeción de los objetivos de la política de salud en México a los planes gubernamentales de cada sexenio complica el establecimiento de estrategias de largo o mediano plazo. Eso es claro cuando se observa que la planeación presupuestal ha seguido la inercia de los gobiernos anteriores, sin alterar las estrategias transexenales - como el Seguro Popular-, ni modificar sus objetivos, de modo que los programas que trascienden los períodos de gobierno aprovechen esa condición para atacar con constancia y consistencia los problemas epidemiológicos del país.

\section{Líneas de acción focalizadas}

En el caso de la mortalidad infantil existen políticas que ya establecen diferencias por estrato socioeconómico en la búsqueda de eficacia, pero no por grupo vulnera-

\footnotetext{
14 Si bien existe la Estrategia Nacional para la Prevención y Control del Sobrepeso, la Obesidad y la Diabetes, pensamos en un plan nacional que no solo sea transversal entre las dependencias del gobierno federal, sino que incluya en él la participación activa de los gobiernos estatales y municipales.
} 
ble. Aunque la mortalidad infantil en general se ha reducido, los datos siguen siendo preocupantes entre los estratos sociales menos favorecidos, las zonas rurales y la población indígena. Dos estados donde hay que prestar atención especial en esta materia son Guerrero y Oaxaca. Una población vulnerable a la que se debe atender especialmente es la indígena. La idea de regionalidad de la cuarta línea de acción general tiene que ver con la incidencia de distintos problemas según la región. Es importante tomar en cuenta que, por la naturaleza del país, no solo la política federal de salud debe establecer diferencias de acción con base en criterios regionales. Todas las políticas públicas nacionales deberían atender tales altibajos o distinciones según la incidencia de los problemas que pretenden atender, a riesgo de que, de no hacerlo, sea imposible solucionar todos los ámbitos de un problema público. El caso de la política de seguridad nacional es uno de los más relevantes como ejemplo del desfase en el diseño de políticas públicas homogéneas, que fracasan en la consecución de sus objetivos porque ignoran la importancia de los factores locales en los problemas públicos.

Siguiendo en la atención al grupo poblacional de los niños, es importante que la política federal de salud enfatice en las acciones de nutrición, de manera que los infantes logren su máximo potencial de desarrollo y disminuyan en lo posible los comportamientos y las condiciones previas a los padecimientos crónico degenerativos. En cuanto a la obesidad, relacionada con la desnutrición, que es la causa de la mayor parte de las enfermedades crónico degenerativas, es importante no perder de vista evidencia clave como el impuesto a bebidas azucaradas, que disminuyó en $6 \%$ el consumo en un año de implementación (Colchero, Popkin, Rivera \& Wen Ng, 2016). Esta disminución no es asunto menor, pues incide en la disminución de problemas de peso y cardiometabólicos (Colchero et al., 2016, p. 1). Otra evidencia clave en materia de política pública es que dicho impuesto tuvo mayor impacto en los hogares de bajo nivel socioeconómico, en los que disminuyeron en promedio $9 \%$ las compras de bebidas azucaradas durante 2014, reducción que alcanzó el 17 \% en diciembre (Colchero et al., 2016). Esta tasa se debe complementar con una política de provisión de alimentos a la población de menos recursos. Aunque los derroteros de esta línea de acción específica son bastante mayores que los que señalamos, la idea central de esta sugerencia es que la imposición de tasas a los alimentos con alto contenido calórico debe tener en cuenta elementos complementarios. Un estudio reciente del Centro de Estudios Económicos de El Colegio de México argumenta que el impuesto a las bebidas azucaradas tuvo tres consecuencias que, por sí mismas, dejan ver la necesidad de acompañar ese tipo de tributación con otras acciones. En primer lugar, tuvo un efecto negativo en el poder adquisitivo de los estratos de ingreso más bajo entre 2012 y 2014; en segundo, tuvo un efecto mínimo en la disminución de la ingesta calórica de la población, y en tercero, disminuyó muy poco el volumen anual de venta de refrescos (Hernández 
Mundo, 2015). Así, las consecuencias en la reducción del consumo —en el caso de bebidas azucaradas - no son suficientes si la única línea de política es el aumento de su precio. Lo urgente es hallar sucedáneos de ingesta calórica para proponer al mercado mexicano que no tengan los efectos negativos de los azúcares y las grasas excesivas. Por tanto, el aspecto tributario es solo un pequeño paso en una estrategia de prevención de gran envergadura cuyo objetivo sea modificar positivamente los hábitos de consumo.

Sobre este mismo tema, es importante mencionar que las compras de bebidas no gravadas aumentaron en promedio $4 \%$ debido principalmente al aumento en la compra de agua embotellada. Esto sugiere que los consumidores están sustituyendo bebidas azucaradas por otras saludables (Colchero et al., 2016). Lo anterior nos lleva a otro tema importante en materia de política pública: el consumo de agua en México. La población mexicana se encuentra entre las que más consumen bebidas azucaradas en el mundo, consumo que representa el $19 \%$ del consumo de energía diario (Popkin, Hernández Cordero, Rivera Dommarco, Barquera, Rodríguez Ramírez \& Villanueva, 2015). La obligatoriedad de instalar bebederos en todas las escuelas de educación básica y media, que entró en vigor en 2014, es ejemplo de las acciones complementarias de las que hemos hablado. Pese a que la instalación de ellos en las escuelas está lejos de completarse, es una medida en el sentido correcto. El consumo de agua en niños es política pública en China, por ejemplo, pues los infantes deben acudir cada dos horas a tomar agua de los bebederos y hacer algunos ejercicios. Esto previene la obesidad y favorece la concentración y el rendimiento intelectual (PRNewswire, 2015). Puesto que el problema de la obesidad afecta a todos los grupos etarios e investigaciones recientes han demostrado que existe relación entre consumo de agua y disminución de triglicéridos en sangre, peso y otros factores de riesgo cardiometabólico (Popkin et al., 2015), la ampliación de la obligatoriedad de la política de bebederos a otros espacios - al menos los de carácter público y negocios del sector formal- podría constituir una de las líneas complementarias, con objetivos de gran calado, que hemos considerado necesarias.

No debemos obviar las consecuencias políticas de los incrementos de impuestos y la estrategia de bebederos. En estos temas existen actores económicos poderosos que se oponen a incrementos impositivos y han logrado influir en la toma de decisiones, principalmente en el Poder Legislativo. Es el caso de las compañías refresqueras, las cuales incluso financian investigación -que curiosamente contradice la idea de que el consumo de refrescos está relacionado con la obesidad, el sobrepeso y la diabetes (Colchero et al., 2016).

En el caso de los jóvenes, como grupo de población, los recursos en materia de salud que les asignan los ramos 12, 50 y 51 del PEF son prácticamente inexistentes. El dato es preocupante si se tiene en cuenta que, como mencionamos, las principales causas de muerte son las relacionadas con las adicciones, como los accidentes 
automovilísticos y los suicidios. En los casos de accidentes y suicidios, el sistema de salud desempeña un papel importante, ya que el $90 \%$ de los accidentes son evitables (Domínguez y Karaisl, 2013, p. 143). Aunque los jóvenes solo representan $9 \%$ de los conductores, este grupo constituye entre $18 \%$ y $30 \%$ de los conductores que mueren en accidentes viales. Para enfrentar esta problemática se sugieren políticas preventivas relacionadas con la regulación de los límites de velocidad, niveles de alcohol, cinturón de seguridad y consumo de drogas. Aunque ya se han instrumentado estas acciones en buena parte del país, no necesariamente ha sido como eslabones de una cadena coherente de política preventiva. Los incentivos negativos pueden ser muy útiles en este sentido. En el Reino Unido, por ejemplo, el seguro de auto es obligatorio y el precio de este en jóvenes es muy alto, casi lo que cuesta adquirir un vehículo. Debido a ello, muy pocos jóvenes manejan automóvil. En México, pese a la magnitud de los costos y a las diversas implicaciones socioeconómicas de este problema, son escasas las políticas públicas cuyo objetivo sea resolver este tipo de problemas.

En el tema del suicidio, la política debe dirigirse al segmento masculino de la población joven pues, como pudimos ver, casi el $90 \%$ de los suicidios en jóvenes corresponden a este sexo. Es importante poner énfasis en la situación de este problema en los estados de Aguascalientes, Quintana Roo y Campeche porque tienen las tasas más altas de suicidio del país, en contraposición a los de Guerrero, Oaxaca y Tlaxcala. En México, más de la mitad de los suicidios son consumados por personas con trastornos depresivos y casi uno de cada cuatro se asocia con el alcoholismo. Dichos padecimientos requieren un adecuado manejo terapéutico y mayor vigilancia cuando se detecta la ideación suicida (INEGI, 2015). De acuerdo con la Organización Mundial de la Salud (oMs), la prevención y el tratamiento adecuado de la depresión, abuso de alcohol y otras sustancias, así como de quienes han intentado suicidarse, permite reducir las tasas de suicidio (INEGI, 2015). En ninguno de los programas del Ramo 12 hay asignación de gasto para prevenir adicciones, $y$ mucho menos problemas como la depresión en jóvenes y adultos.

Tanto para la población adulta como para la de adultos mayores se requieren políticas públicas dirigidas a prevenir la diabetes. Como pudimos ver, en los ramos 12,50 y 51 no hay programas que tengan este objetivo específico. La gravedad de la epidemia de diabetes y el hecho de que se trata de una enfermedad prevenible permiten sostener el argumento principal de este artículo. Su impacto sobre la calidad de vida de las personas que la padecen la convierte en un área prioritaria para el sector salud. En este sentido, es importante tomar en cuenta que es mucho mayor el problema en algunas entidades: Distrito Federal, Nuevo León, Veracruz y Estado de México. Para diseñar un esquema de política pública adecuado es necesario identificar por qué en ellas son tan elevadas las tasas, es decir, a qué clase de comportamientos y hábitos de consumo responden los altos índices de prevalencia. De entrada, es importante impulsar la creación de políticas públicas que incidan en 
la erradicación de los estilos de vida asociados con el desarrollo de diabetes, como pueden ser aquellas encaminadas a disminuir el consumo de bebidas azucaradas y las que favorezcan una alimentación adecuada. Algunas estimaciones indican que, por ejemplo, en Estados Unidos desde 1997 se destina más de $15 \%$ del gasto en salud para atender a los diabéticos. En México, son muy variables las estimaciones de costos de atención por paciente, pues van de 700 a 3,200 dólares anuales, lo que significa de $5 \%$ a $14 \%$ del gasto en salud.

En términos presupuestales, en 2012 el gasto para el manejo de la diabetes fue de más de 4,524 millones de dólares en los pacientes que reportan estar acudiendo a atención, 15 \% más que la cifra estimada para 2011. Para contextualizarla, este monto es superior a los 3,790 millones de dólares de recursos asignados al Seguro Popular en 2010 (Hernández Ávila, Gutiérrez, Reynoso Noverón \& Reynoso Noverón, 2013). ¿Qué quiere decir esto? Que en el año 2050, dadas las tendencias epidemiológicas y las proyecciones demográficas, será imposible atender a los pacientes con este padecimiento. Por ello la prevención es la única salida en este problema.

Las adicciones, especialmente el alcohol, también tienen que ser objeto de política pública entre la población adulta, ya que se relaciona con la mayoría de las causales de mortandad que hemos señalado. Los impuestos al consumo, las campañas informativas, el límite al consumo y, obviamente, el gasto asignado a este problema deben ser prioridad. Actualmente, en los ramos analizados este tema no es prioritario. Existe evidencia que debemos tomar en cuenta como referencia para el diseño de políticas impositivas al alcohol, como ya ocurre con el tabaco, en que gracias a campañas de información y prohibiciones de consumo, ha sido posible disminuir el tabaquismo. Al respecto, los estudios demuestran que la manera más efectiva de reducir el consumo de tabaco ha sido el aumento de su precio mediante incrementos impositivos. Los precios elevados desalientan el inicio de los jóvenes en el consumo de cigarrillos y alientan a quienes ya fuman a dejar de hacerlo. En México, el consumo de cigarrillos ha disminuido conforme aumentaba su precio (Waters, Sáenz de Miera, Ross \& Reynales Shigematsu, 2010).

Concretamente, los estudios recomiendan incrementar significativamente los impuestos al consumo de modo que el impuesto total (Impuesto Especial sobre Producción y Servicios, IEPS, más Impuesto al Valor Agregado, IVA) represente el $75 \%$ del precio final, lo cual es compatible con las mejores prácticas internacionales y característico de países con políticas de control del tabaco exitosas. También recomiendan aumentar la dependencia de los impuestos específicos al tabaco por sobre los impuestos según el valor; estos son más sencillos de administrar y tienden a reducir la diferencia de precio entre las marcas, lo que contrarresta la tendencia a optar por cigarrillos más baratos cuando los impuestos se incrementan. En tercer lugar, ajustar los impuestos específicos conforme a la inflación, de lo contrario con el tiempo se deprecia el valor de los impuestos. En cuarto lugar, reforzar la adminis- 
tración del impuesto al tabaco; una administración exitosa del mismo dependerá del registro y la autorización de todos los productores comerciales, importadores y vendedores mayoristas y minoristas. Finalmente, considerar el uso de parte de los ingresos generados por el aumento de impuestos al tabaco para financiar iniciativas de salud pública destinadas a reducir su consumo, financiar otros servicios de salud y fortalecer los mecanismos para combatir el comercio ilegal de productos de tabaco (Waters et al., 2010).

En tal sentido, no debemos olvidar la importancia de proporcionar información al consumidor. Es muy probable que gran parte de las razones por las cuales se consumen en exceso alcohol y comida chatarra sea la asimetría de información que padecen los consumidores. Al no saber claramente cuáles son los componentes de estos productos ni los efectos secundarios de su consumo, este se realiza sin control y en exceso, lo que genera malos hábitos y, a la postre, afecta la salud. Para ello, tomemos como ejemplo las campañas contra el consumo de cigarros en México, que están plasmadas en imágenes y texto en la propia caja del producto.

Si bien las líneas de acción focalizadas se engarzan con las estrategias transversales que se proponen, su componente de especificidad no es trivial, pues una estrategia integral debe tener en cuenta que la prevención requiere de frentes de acción simultáneos.

\section{Consideraciones finales}

La intención principal de este artículo ha sido llamar la atención sobre un problema que atenta contra la estabilidad del sistema de salud y las finanzas públicas mexicanas. La lógica de la política pública ofrece elementos para solucionar los problemas públicos. En el caso de la realidad epidemiológica del país, las previsiones ponen sobre la mesa con antelación suficiente los principales detalles de un problema de proliferación de padecimientos crónico degenerativos. En pocas ocasiones es posible prever la emergencia de un problema público. Por eso, en el caso que analizamos, es oportuno proponer líneas de acción y, en general, la articulación de una política pública federal que intente detener la evolución de las condiciones actuales al estadio epidémico, lo cual podría suceder en el mediano y largo plazos.

A partir del análisis y las propuestas de política anteriores es posible redondear la discusión sobre algunos argumentos que, más allá de reafirmar los fines de este artículo, quedan como líneas de discusión para investigaciones futuras. No hemos limitado la discusión a las consecuencias políticas, sociales o financieras de las proyecciones del problema en el mediano plazo. La discusión enfatizó las consecuencias médicas, es decir, intentó caracterizar la evolución que tendrá el perfil epidemiológico del país en algunas décadas pues, más que nada, tales consideraciones tienen 
la fortaleza de la ciencias fisiológicas, de modo que hemos intentado que nuestras propuestas de hipótesis se fortalezcan con evidencia que carece de subjetividad.

La discusión que hemos dado se resume en una idea: la única forma en que la política pública y el diseño presupuestal federal pueden incidir positivamente en las previsiones epidemiológicas del país es mediante un cambio de paradigma, un énfasis en la prevención de las enfermedades crónico degenerativas como gasto de inversión.

\section{Referencias}

Aguilar, L. F. (2010). Introducción. En Aguilar, L. F. (comp.) (2010), Política pública (pp. 1760). México: Escuela de Administración Pública de la Ciudad de México.

Borges, G., Orozco, R. \& Medina Mora, M. E. (2012). Índice de riesgo para el intento suicida en México. Salud Pública de México, 54(6), 595-606.

Campos Nonato, I., Barquera, S. \& Aguilar, C. (2012). Efecto de la obesidad sobre otras enfermedades crónicas y la mortalidad. En Rivera Dommarco, J. A., Hernández Ávila, M., Aguilar Salinas, C. A., Vadillo Ortega, F. \& Murayama Rendón, C. (eds.), Obesidad en México. Recomendaciones para una política de Estado. México: Universidad Nacional Autónoma de México.

Colchero, A., Popkin, B. M., Rivera, J. A. \& Wen Ng, S. (2016). Beverage purchases from stores in Mexico under the excise tax on sugar sweetened beverages: observational study. British Medical Journal, 16(352), 1-9.

Consejo Nacional de Población (2012). Mortalidad. Principales causas de muerte 2012. Recuperado de http://www.conapo.gob.mx/es/CONAPO/Mortalidad

Cordero Hernández, A. \& Pinto Almazán, R. (2014). Diabetes mellitus tipo 1 y 2. Estudio epidemiológico del primer año de servicio de Consulta Externa del Hospital Regional de Alta Especialidad de Ixtapaluca. Evidencia Médica e Investigación en Salud, 7(1), 10-18.

Cuevas Nasu, L., Rivera Dommarco, J. A., Shamah Levy, T., Mundo Rosas, V. \& Méndez Gómez Humarán, I. (2014). Inseguridad alimentaria y estado de nutrición en menores de cinco años de edad en México. Salud Pública de México, 56(1), 547-554.

Domínguez, C. \& Karaisl, M. (2013). Más allá del costo a nivel macro: los accidentes viales en México, sus implicaciones socioeconómicas y algunas recomendaciones de política pública. Revista Legislativa de Estudios Sociales y de Opinión Pública, 6(12), 131-148.

Encuesta Nacional de Adicciones 2011. Alcohol. Recuperado de http://www.conadic.salud. gob.mx/pdfs/ENA_2011_ALCOHOL.pdf

Encuesta Nacional de Salud y Nutrición 2012. Resultados nacionales. Recuperado de http:// ensanut.insp.mx/informes/ENSANUT2012ResultadosNacionales.pdf

González Guzmán, R. \& Alcalá Ramírez, J. (2006). Consumo de alcohol y salud pública. Revista de la Facultad de Medicina, 49(6), 238-241. 
Guerrero López, C.M., Muños Hernández, J. A., Sáenz de Miera Juárez, B., Pérez Núñez, R. \& Reynales Shigematsu, L. M. (2013). Impacto del consumo nocivo del alcohol en accidentes y enfermedades crónicas en México. Salud Pública de México, 55(2), 282-288.

Gutiérrez, G., Autrique, M. \& Ceballos, G. (2012). Sobrepeso y obesidad: frecuentes características del estado nutricional en una muestra de pacientes mexicanas con cáncer de mama localmente avanzado. Revista Colombiana de Cancerología, 16(3), 187-191.

Hernández Ávila, M., Gutiérrez, M., Reynoso Noverón, J. P. \& Reynoso Noverón, N. (2013). Diabetes mellitus en México. El estado de la epidemia. Salud Pública de México, 55(2), 129-136.

Hernández Mundo, K. (2015, 9 de diciembre). El IEPs calórico tiene impacto limitado en consumo: Colmex. El Economista.

Informe sobre la situación mundial de las enfermedades no transmisibles 2010 (2011). Resumen de orientación. oms. Recuperado de http://www.who.int/nmh/publications/ ncd_report_summary_es.pdf

Instituto Nacional de Estadística y Geografía (INEgi) (2014). Perfil sociodemográfico de jóvenes. Recuperado de http://www.inegi.org.mx/prod_serv/contenidos/espanol/bvinegi/productos/censos/poblacion/2010/perfil_socio/jovenes/702825056636.pdf

Instituto Nacional de Estadística y Geografía (INEGi) (2014). Perfil sociodemográfico de niños. Recuperado de http://www.inegi.org.mx/prod_serv/contenidos/espanol/bvinegi/productos/censos/poblacion/2010/perfil_socio/ninos/702825056629.pdf

Instituto Nacional de Estadística y Geografía (INEGI) (2015). Perfil sociodemográfico. Estados Unidos Mexicanos. Recuperado de http://www.inegi.org.mx/prod_serv/ contenidos/espanol/bvinegi/productos/censos/poblacion/2010/perfil_socio/ uem/702825047610_1.pdf

Instituto Nacional de Estadística y Geografía (INEGI) (2015). Estadísticas sobre suicidio en México. Recuperado de http://www.inegi.org.mx/saladeprensa/aproposito/2015/ suicidio0.pdf

Lozano, R. et al. (2013). La carga de enfermedades, lesiones, factores de riesgo y desafíos para el sistema de salud en México. Salud Pública de México, 55(6), 580-595.

Manrique Espinoza, B., Salinas Rodríguez, A., Moreno Tamayo, K. M., Acosta Castillo, I. \& Sosa Ortiz, A. L. (2013). Condiciones de salud y estado funcional de los adultos mayores en México. Salud Pública de México, 55(2), 323-331.

Masud Yunes Zárraga, J. L., Barrios Reyes, A. \& Duarte Ortuño, A. (2009). Prevalencia de desnutrición, sobrepeso y obesidad en niños del CENDI. Pediatría de México, 11(2), 62-66.

Maya Bautista, J. E. (2012). El cambio demográfico en México. Centro de Investigación Económica y Presupuestaria (CIEP). Recuperado de http://ciep.mx/el-cambio-demografico-en-mexico/

Mondragón, L., Borges, G. \& Gutiérrez, R. (2001). La medición de la conducta suicida en México: estimaciones y procedimientos. Salud Mental, 24(6), 4-15. 
Muradás, M. C., Solís, A. \& Sánchez Castillo, M. (2014). Ganancias y pérdidas de la esperanza de vida por enfermedades relacionadas con el sobrepeso y la obesidad. La situación demográfica de México 2014, 1-34. Recuperado de http://www.conapo.gob.mx/ work/models/CONAPO/Resource/2414/1/images/04_Muradas_Solis_Sanchez.pdf

Organización para la Cooperación el Desarrollo Económicos (ocDE) (2015). Estadísticas de la OCDE sobre la salud 2014. México en comparación. Recuperado de http://www. oecd.org/els/health-systems/Briefing-Note-MEXICO-2014-in-Spanish.pdf

Organización para la Cooperación el Desarrollo Económicos (OCDE) (2016). Estudios de la oCDE sobre los sistemas de salud: México 2016. Recuperado de http://funsalud.org. mx/portal/wp-content/uploads/2016/01/ocde_rhsmx_sp.pdf

Organización Panamericana de la Salud (ops) (2012). Salud en las Américas. Capítulo México. México: ops.

Pons, J. \& Berjano, E. (1999). El consumo abusivo de alcohol en la adolescencia: un modelo explicativo desde la psicología social. Valencia: Plan Nacional sobre Drogas.

Popkin, B., Hernández Cordero, S., Rivera Dommarco, J., Barquera, S., Rodríguez Ramírez, S. \& Villanueva, M. A. (2014). La sustitución de bebidas azucaradas por agua podría reducir riesgo de síndrome metabólico en mujeres mexicanas obesas. The Journal of Nutrition. Recuperado de http://jn.nutrition.org/content/early/2014/09/03/ jn.114.193490.full.pdf + html

PRNewswire (2015, agosto). Regresan los bebederos a México. Especialistas promueven su uso para combatir la obesidad a través de la hidratación. Recuperado de http:// www.prnewswire.com/news-releases/regresan-los-bebederos-a-mexico-especialistas-promueven-su-uso-para-combatir-a-la-obesidad-a-traves-de-la-hidratacion-520775211.html

Ramo 11 de la base de datos sobre programas presupuestarios 2010-2015. SHCP.

Ramo 12 de la base de datos sobre programas presupuestarios 2010-2015. SHCP.

Ramo 20 de la base de datos sobre programas presupuestarios 2010-2015. sHCP.

Ramo 50 de la base de datos sobre programas presupuestarios 2010-2015. sHCP.

Ramo 51 de la base de datos sobre programas presupuestarios 2010-2015. sHCP.

Reglas de operación de los programas presupuestales publicados en el Diario Oficial de la Federación (DOF) para los ramos 12, 50 y 51.

Saldívar Cerón, E. I., Garmendia Ramírez, A., Rocha Acevedo, M. A. \& Pérez Rodríguez, P. (2015). Obesidad infantil: factor de riesgo para desarrollar pie plano. Boletín Médico del Hospital Infantil de México, 72(1), 55-60.

Shannah Levy, T., Amaya Castellanos, M. A. \& Cuevas Nasu, L. (2015). Desnutrición y obesidad: doble carga en México. Revista Digital Universitaria, 16(5).

Shannah-Levy, T., Mundo Rosas, V. \& Rivera Dommarco, J. A. (2014). La magnitud de la inseguridad alimentaria en México: su relación con el estado de nutrición y con factores socioeconómicos. Salud Pública de México, 56(1), 579-585.

Torres Sánchez, L. E., Rojas Martínez, R., Escamilla Núñez, C., Vara Salazar, E. \& Lazcano 
Ponce, E. (2014). Tendencias en la mortalidad por cáncer en México de 1980 a 2011. Salud Pública de México, 56(5), 473-492.

Waters, H., Sáenz de Miera, B., Ross, H. \& Reynales Shigematsu, L. M. (2010). La economía del tabaco y los impuestos al tabaco en México. Bloomberg Philantropies y Bill and Melinda Gates Foundation. 